\title{
313 NEW ASTEROID ROTATION PERIODS FROM PALOMAR TRANSIENT FACTORY OBSERVATIONS
}

\author{
Chan-Kao Chang ${ }^{1}$, Wing-Huen Ip ${ }^{1,2}$, Hsing-Wen Lin ${ }^{1}$, Yu-Chi Cheng ${ }^{1}$, Chow-Choong Ngeow ${ }^{1}$, Ting-Chang Yang $^{1}$, \\ Adam Waszczak ${ }^{3}$, Shrinivas R. Kulkarni ${ }^{4}$, David Levitan ${ }^{4}$, Branimir Sesar ${ }^{4}$, Russ Laher ${ }^{5}$, \\ Jason Surace 5 , Thomas. A. Prince ${ }^{4}$, and the PTF Team \\ ${ }^{1}$ Institute of Astronomy, National Central University, Jhongli, Taiwan; rex@astro.ncu.edu.tw \\ ${ }^{2}$ Space Science Institute, Macau University of Science and Technology, Macau, China \\ ${ }^{3}$ Division of Geological and Planetary Sciences, California Institute of Technology, Pasadena, CA 91125, USA \\ ${ }^{4}$ Division of Physics, Mathematics and Astronomy, California Institute of Technology, Pasadena, CA 91125, USA \\ ${ }^{5}$ Spitzer Science Center, California Institute of Technology, M/S 314-6, Pasadena, CA 91125, USA \\ Received 2014 February 14; accepted 2014 April 17; published 2014 May 16
}

\begin{abstract}
A new asteroid rotation period survey has been carried out by using the Palomar Transient Factory (PTF). Twelve consecutive PTF fields, which covered an area of $87 \mathrm{deg}^{2}$ in the ecliptic plane, were observed in the $R$ band with a cadence of $\sim 20$ minutes during 2013 February 15-18. We detected 2500 known asteroids with a diameter range of $0.5 \mathrm{~km} \leqslant D \leqslant 200 \mathrm{~km}$. Of these, 313 objects had highly reliable rotation periods and exhibited the "spin barrier" at $\sim 2 \mathrm{hr}$. In contrast to the flat spin-rate distribution of the asteroids with $3 \mathrm{~km} \leqslant D \leqslant 15 \mathrm{~km}$ shown by Pravec et al., our results deviated somewhat from a Maxwellian distribution and showed a decrease at the spin rate greater than $5 \mathrm{rev} \mathrm{day}^{-1}$. One superfast rotator candidate and two possible binary asteroids were also found in this work.
\end{abstract}

Key words: minor planets, asteroids: general - surveys

Online-only material: color figures, machine-readable table

\section{INTRODUCTION}

Time-series photometry is a powerful tool to derive physical properties of solar system objects including the rotation periods and shapes of asteroids and cometary nuclei. Harris (1996) showed a "spin barrier" at $2.2 \mathrm{hr}$ for asteroids with $D \gtrsim$ $1 \mathrm{~km}$, which indicates that large asteroids are gravitationally bounded aggregations (i.e., rubble-pile structure). Following that study, Pravec \& Harris (2000) revealed that the asteroids with diameters larger than a few hundred meters are rubble piles and have spin rates lower than the "spin barrier, whereas smaller asteroids may rotate faster than the "spin barrier" (i.e., superfast rotator; see an example study in Hergenrother \& Whiteley 2011) and are likely monolithic objects. Exceptions to this rule are rare. For example, 2001 OE84 has a diameter of $0.9 \mathrm{~km}$ and a rotation period of 29.19 minutes (Pravec et al. 2002). Subsequently, Holsapple (2007) suggested a sizedependent strength for asteroids and predicted the existence of kilometer-sized superfast rotators. Moreover, the spin-rate distributions of differently sized asteroids are useful to investigate how different mechanisms alter the asteroid rotations. Salo (1987) showed that a collisionally evolved asteroidal system should have a Maxwellian spin-rate distribution. This is true for asteroids with $D>40 \mathrm{~km}$ (Pravec et al. 2002). However, mechanisms in addition to collision, in particular the Yarkovsky-O'Keefe-Radzievskii-Paddack (YORP; Rubincam 2000), create excesses at both the very slow and fast ends for smaller size asteroids (Pravec et al. 2002; Polishook \& Brosch 2008; Masiero et al. 2009).

While this information is of importance in establishing the global property of the asteroids, only a small fraction ( $\sim 4000$ among $\sim 620,000$ known asteroids) have published light curves and rotation periods (Warner et al. 2009). In recent years, large field-of-view surveys have been used to study asteroid rotation periods (see examples of Masiero et al. 2009; Polishook et al. 2012). With the increasing volume of such data sets, the studies of the asteroid rotation properties (i.e., spin-rate limit and spin-rate distribution) can be done as a function of different taxonomic types, dynamical groups, and asteroid families.

As demonstrated by the pilot work of Polishook et al. (2012), the asteroidal observations in the Palomar Transient Factory (PTF) synoptic survey could make important contributions in this respect. They reported their analysis of four overlapping PTF fields covering $21 \mathrm{deg}^{2}$ with multiple observations $(\geqslant 10$ images) per night and a typical cadence of $\sim 20 \mathrm{~min}-$ utes. Of the 624 asteroids detected in their work, 88 of them have well-determined rotation periods and 85 have low-quality rotation periods. Here, we continue this line of work by presenting 312 new, high-quality, asteroid rotation periods. The final goal of this long-term project is to collect a sample of about $\sim 10^{4}$ asteroid rotation periods.

In Section 2, the observation information is described. The method of data analysis is given in Section 3. The results of the statistical distribution of the rotation periods and the properties of some individual asteroids are presented in Section 4. A summary and conclusion can be found in Section 5 .

\section{OBSERVATIONS}

The $\mathrm{PTF}^{6}$ is a synoptic survey designed to explore the transient and variable sky (Law et al. 2009; Rau et al. 2009), which employs the 48 inch Oschin Schmidt Telescope equipped with an 11-chip mosaic CCD camera (i.e., the former CFHT-12K camera, in which the chip no. 3 is out of function). The available filters include Mould- $R$, Gunn- $g^{\prime}$, and $\mathrm{H} \alpha$. Such a configuration has a field of view of $\sim 7.26 \mathrm{deg}^{2}$ and a pixel scale of $1^{\prime \prime} .01$. The $5 \sigma$ median limiting magnitude of an exposure of $60 \mathrm{~s}$ in the $R$ band is $\sim 21 \mathrm{mag}$ (Law et al. 2010).

As part of the Ten Thousand Asteroid Rotation Periods project (10kARPs), 12 consecutive PTF fields, which covered an area of $87 \mathrm{deg}^{2}$ on the ecliptic plane, were observed in the $R$ band with a cadence of $\sim 20$ minutes during 2013 February $15-18$. The exposure time of each image was $60 \mathrm{~s}$. Figure 1

\footnotetext{
6 http://ptf.caltech.edu
} 
Table 1

\begin{tabular}{lccccccc}
\multicolumn{7}{c}{ Observation Information } \\
\hline \hline Field ID & R.A. & Decl. & {$[l, b]$} & Feb 15 & Feb 16 & Feb 17 & Feb 18 \\
& $\left({ }^{\circ}\right)$ & $\left({ }^{\circ}\right)$ & $\left({ }^{\circ}\right)$ & $\Delta t, N_{\exp }$ & $\Delta t, N_{\exp }$ & $\Delta t, N_{\exp }$ & $\Delta t, N_{\exp }$ \\
\hline 3654 & 101.02 & 21.38 & {$[192.97,8.02]$} & $3.4,10$ & $5.1,16$ & $6.8,19$ & $6.4,19$ \\
3655 & 104.69 & 21.38 & {$[194.47,11.11]$} & $3.4,10$ & $5.3,15$ & $6.8,19$ & $6.4,19$ \\
3656 & 108.37 & 21.38 & {$[195.92,14.23]$} & $3.4,10$ & $5.3,15$ & $7.2,20$ & $6.7,20$ \\
3657 & 112.04 & 21.38 & {$[197.33,17.36]$} & $3.4,10$ & $5.4,15$ & $7.2,20$ & $7.1,21$ \\
3658 & 115.71 & 21.38 & {$[198.72,20.52]$} & $3.4,10$ & $5.5,16$ & $7.6,21$ & $7.4,22$ \\
3749 & 90.93 & 23.62 & {$[186.63,0.81]$} & $3.4,10$ & $5.3,14$ & $6.4,18$ & $6.0,18$ \\
3750 & 94.64 & 23.62 & {$[188.27,3.79]$} & $3.4,10$ & $5.1,15$ & $6.4,18$ & $6.0,18$ \\
3751 & 98.35 & 23.62 & {$[189.84,6.81]$} & $3.4,10$ & $5.1,15$ & $6.8,19$ & $6.4,19$ \\
3752 & 102.06 & 23.62 & {$[191.34,9.87]$} & $3.4,10$ & $5.0,14$ & $6.8,19$ & $6.7,20$ \\
3753 & 105.77 & 23.62 & {$[192.80,12.95]$} & $3.4,10$ & $5.1,15$ & $7.2,20$ & $6.7,20$ \\
3754 & 109.48 & 23.62 & {$[194.21,16.07]$} & $3.4,10$ & $5.1,15$ & $7.6,21$ & $7.1,21$ \\
3755 & 113.20 & 23.62 & {$[195.58,19.21]$} & $3.4,10$ & $5.4,15$ & $7.6,21$ & $7.4,22$ \\
\hline
\end{tabular}

Note. $\Delta t$ is the time duration spanned by each observing set in hours and $N_{\exp }$ is the total number of exposures for each night and field.

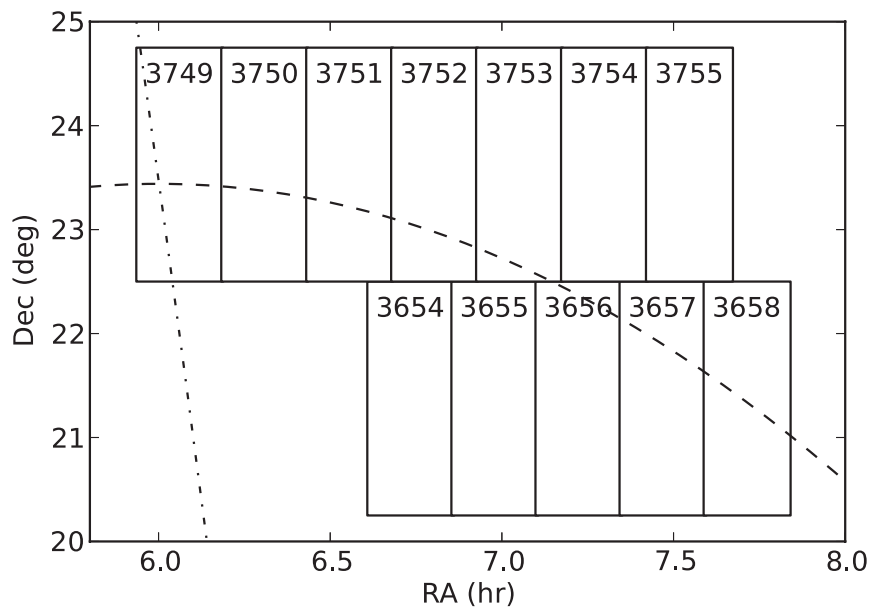

Figure 1. Configuration of $12 \mathrm{PTF}$ fields. Each rectangle represents a PTF field with a field ID on top. The field of view of each PTF field is $3.50 \times 2.31 \sim$ $7.26 \mathrm{deg}^{2}$. The dashed and dot-dashed lines show the ecliptic and Galactic planes, respectively. Note that the scales of R.A. and decl. are not in proper ratio.

shows the field configuration and Table 1 lists the observation information. Because this campaign was dedicated to the study of Galactic variables as well, our target fields were close to the Galactic plane.

\section{DATA ANALYSIS}

\subsection{Data Reduction and Photometry Calibration}

Each PTF exposure was processed by the PTF photometric pipeline, which included image splitting, debiasing, flatfielding, generation of mask images, source extraction, astrometric calibration, and photometric calibration (Grillmair et al. 2010; Laher et al. 2014). The final products of this pipeline included reduced images, mask images, and source catalogs. The absolute photometric calibration, described in Ofek et al. (2012a, 2012b), was done by using Sloan Digital Sky Survey (SDSS) stars (York et al. 2000) and routinely reached a precision of $\sim 0.02 \mathrm{mag}$. In this work, we used source catalogs computed by SEXtractor (Bertin \& Arnouts 1996) to extract asteroid light curves and employed relative (light-curve-calibrated) photometry (for algorithm details, see Levitan et al. 2011; Ofek et al. 2012a), which typically had a relative photometry accu- racy of $\sim 3$ mmag and $\sim 0.1$ mag in the bright $(\sim 15 \mathrm{mag})$ and faint (i.e., $\sim 19 \mathrm{mag}$ ) ends, respectively (Agüeros et al. 2011; Levitan et al. 2011; Ofek et al. 2011). The photometric calibration described above was done on night, field, and CCD bases. Therefore, a systemic offset was introduced to each data set obtained from different CCDs, fields, and nights. This small offset will be corrected in the period-fitting process, later described in Section 3.4.

\subsection{Light-curve Extraction of Known Asteroids}

The detections in a PTF source catalog were divided into stationary sources (i.e., the source would be detected at the same position repeatedly) and nonstationary sources (e.g., moving objects and false detections). To rule out the detections of stationary sources in the following asteroid light-curve extraction, we performed a spatial cross-match with a radius of $1^{\prime \prime}$ on each source catalog against the reference source catalog. To build the reference source catalog, we used three PTF source catalogs with the best seeing of each night to pick out the sources that had been detected more than three times in the same position (i.e., within $1^{\prime \prime}$ radius). The mean position of the detections of the same reference source was assigned to be its R.A. and decl. Then, we performed another spatial crossmatch with a radius of $1^{\prime \prime}$ on the detections of nonstationary sources of each source catalog against the ephemerides of the asteroids with $V \leqslant 22 \mathrm{mag}$. The asteroidal ephemerides were obtained from MPChecker ${ }^{7}$ according to our exposures. In the last step, the detections from the same asteroid were combined to generate its light curve. When a light curve contained more than five detections, it was identified as a real event (hereafter, the PTF-detected asteroids).

\subsection{Photometric Stability Evaluation}

The photometric stability of each source catalog was also evaluated. To do this, we chose the reference sources in the $1718 R$ magnitude range that had standard deviations of $\leqslant 0.075$ mag during the whole campaign (i.e., relatively brighter sources without brightness variations) to be the photometric reference stars (hereafter, photo-ref-stars). Then, we grouped the photo-ref-stars into bins of $0.1 \mathrm{mag}$ and calculate their mean

\footnotetext{
7 The online ephemerides service hosted at the Minor Planet Center; http://scully.cfa.harvard.edu/cgi-bin/checkmp.cgi.
} 


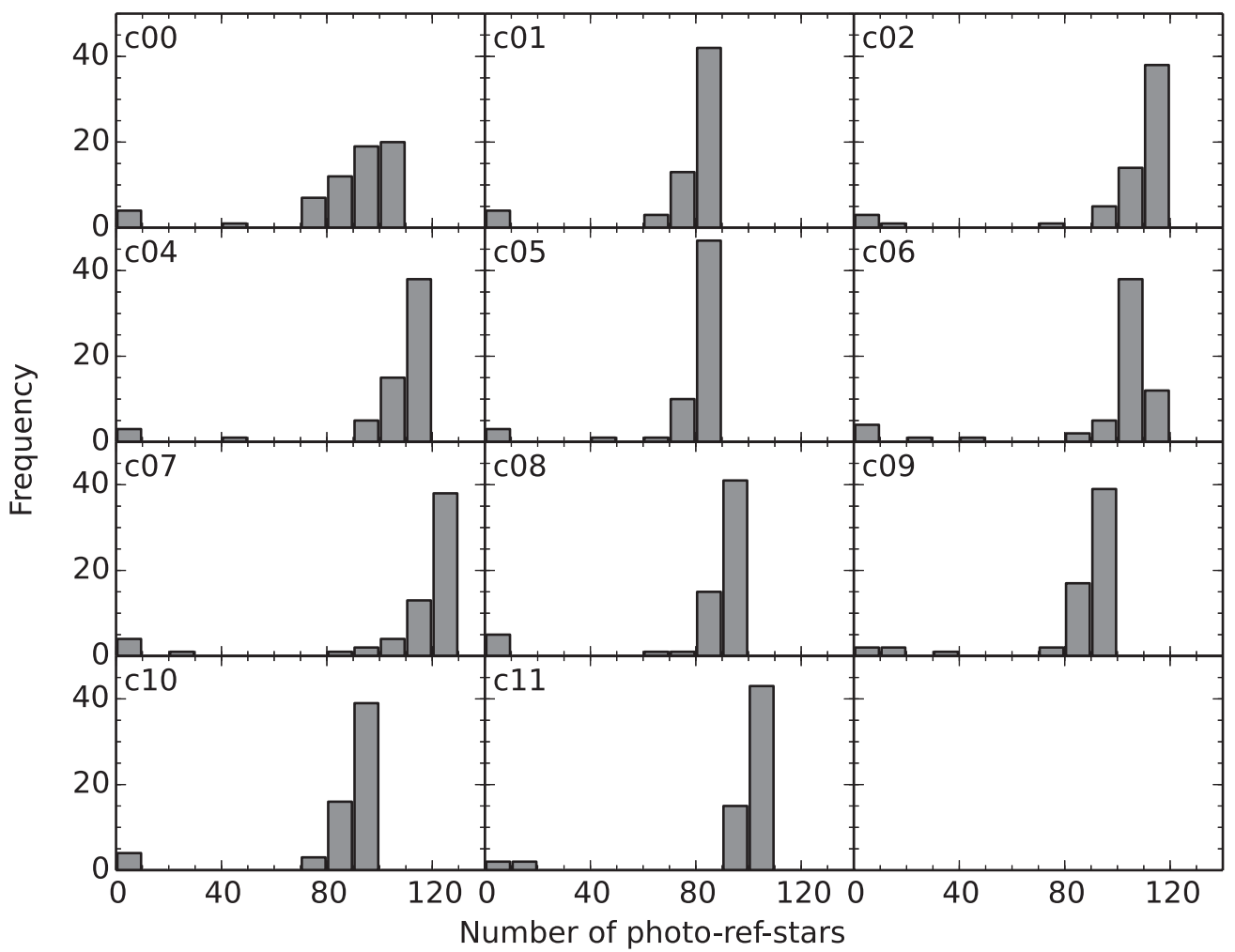

Figure 2. The number distribution of photo-ref-stars of $17.5<R<17.6$ mag for each CCD in field 3655. The CCD numbers are shown on the upper-left corner of each plot.

magnitudes and standard deviations for each source catalog. To judge the photometric stability of each source catalog, we only considered those bins that had more than 30 photo-ref-stars. When a source catalog had one of the following conditions, it would be identified as photometrically unstable and not to be used in the asteroid rotation period analysis: (1) three or more bins had mean magnitudes out of the bin boundaries, (2) three or more bins had standard deviations of $\geqslant 0.075 \mathrm{mag}$, and (3) six or more bins had less than 30 photo-ref-stars. In general, most source catalogs (i.e., $\sim 90 \%$ ) could fulfill the requirement described above. The rest failing to pass the criteria were mostly due to the bad weather exposures, which usually made the three conditions happen at the same time. Figure 2 shows the number distributions of the photo-ref-stars of $17.5<\operatorname{mag}<$ 17.6 of all source catalogs for each CCD in field 3655. Most catalogs had more than 80 photo-ref-stars, and only several catalogs had less than 30 . We also excluded the detections that were flagged by the PTF photometric pipeline as artifacts (e.g., aircraft/satellite track, high dark current pixel, noisy/hot pixel, saturated pixel, dead/bad pixel, ghost image, dirt on the optics, CCD-bleed, or bright star halo, and the defects flagged by the SExtractor).

\subsection{Rotation Period Analysis}

For measuring the synodic rotation periods of the PTF-detected asteroids, the observing times were corrected for light-travel time (i.e., the time interval of a photon traveling from object to Earth), and the magnitudes were reduced to both heliocentric $(r)$ and geocentric $\triangle$ distances at 1 AU by

$$
M_{R(r=1, \triangle=1)}=R+5 \log (r \triangle)
$$

where $M_{R}$ is the $R$-band reduced magnitude. The orbital elements were obtained from the Minor Planet Center ${ }^{8}$, and the heliocentric and geocentric distances were calculated by the PyEphem. 9

Because the phase angles $(\alpha)$ only had a small change in a campaign over four nights, we applied the $H G$ system with a fixed $G_{R}$ slope of 0.15 to estimate the absolute magnitude $H_{R}$ (Bowell et al. 1989):

$$
H_{R}=\left\langle M_{R(r=1, \triangle=1)}\right\rangle+2.5 \log \left[\left(1-G_{R}\right) \phi_{1}+G_{R} \phi_{2}\right],
$$

where $\phi_{1}$ and $\phi_{2}$ are the phase angle parameters as below:

$$
\begin{aligned}
& \phi_{1}=\exp \left[-3.33 \tan (0.5\langle\alpha\rangle)^{0.63}\right], \\
& \phi_{2}=\exp \left[-1.87 \tan (0.5\langle\alpha\rangle)^{1.22}\right] .
\end{aligned}
$$

Then, we fitted a second-order Fourier series to each asteroid light curve that had more than eight detections to search the periodicity:

$M_{i, j}=\sum_{k=1,2}^{N_{k}} B_{k} \sin \left[\frac{2 \pi k}{P}\left(t_{j}-t_{0}\right)\right]+C_{k} \cos \left[\frac{2 \pi k}{P}\left(t_{j}-t_{0}\right)\right]+Z_{i}$,

where $M_{i, j}$ is the $R$-band reduced magnitude measured at the light-travel time-corrected epoch $t_{j}, B_{k}$, and $C_{k}$ are the Fourier coefficients, $P$ is the rotation period, and $t_{0}$ is an arbitrary epoch. As described in Section 3.1, the photometric calibration was carried out on night, field, and CCD bases. Thus, we also

\footnotetext{
8 http://minorplanetcenter.net

9 http://rhodesmill.org/pyephem/
} 


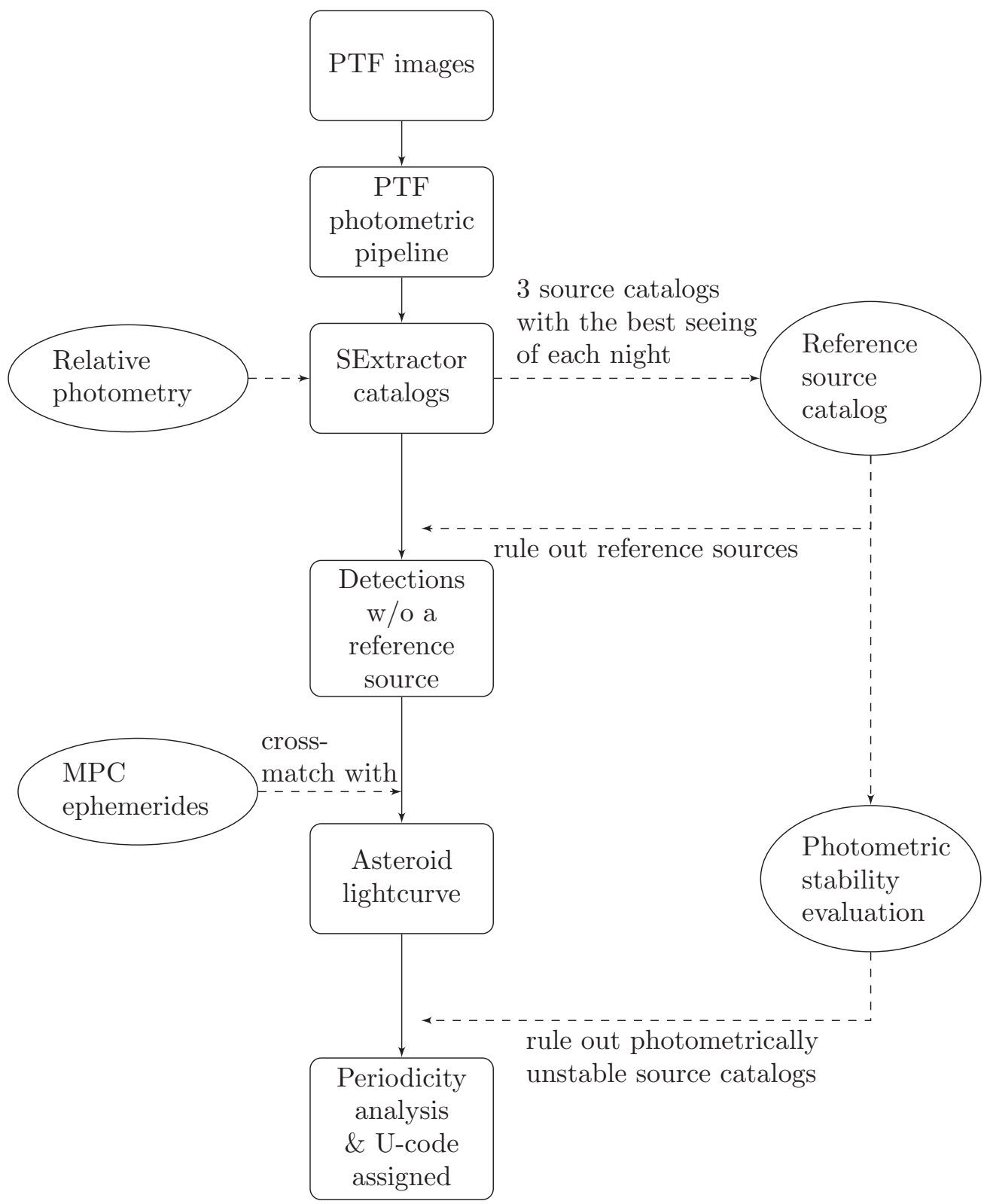

Figure 3. Data process flow chart. See Section 3 for explanation.

fitted a constant value $Z_{i}$ in Equation (5) to correct the small systematic offsets between different data sets, where a data set was defined as all the measurements taken on the same night, field, and CCD with the subscript $i$ marking the $i$ th data set. The Equation(5) was solved by using least-squares minimization for each given $P$ to obtain the other free parameters. We tried the $0.25-50$ frequency range with a step of 0.0025 to cover the majority of asteroid rotation periods (e.g., about 20 minutes to about 80 hours; Pravec \& Harris 2000). Then, we reviewed all possible rotation periods (i.e., the periods with outstanding $\chi^{2}$ values from the others) for each object by inspecting the folded light curves to pick out the best result and assigned a quality code $U$ as introduced by Warner et al. (2009, where 3 means highly reliable, 2 means some ambiguity, 1 means possible but may be wrong, and 0 means no detection). To estimate the uncertainty of the derived rotation period, we calculated the range of periods with $\chi^{2}$ smaller than $\chi_{\text {best }}^{2}+\Delta \chi^{2}$, where $\chi_{\text {best }}^{2}$ is the $\chi^{2}$ of the pick-out period and $\Delta \chi^{2}$ is calculated from the inverse $\chi^{2}$ distribution assuming $1+2 N_{k}+N_{s}$ degrees of freedom. The amplitudes of the objects with full light-curve coverage were adopted from the second-order Fourier series fitting. However, this would probably underestimate the amplitudes of the light curves of sharp minimum/maximum. Some folded light curves just covered part of a rotation period, and some only showed a single minimum due to their sparse data points; however, we still assigned them $U=2$ for their clear folded light curves. To give lower limits on the amplitude for these objects, we calculated a $90 \%$ magnitude range centered on the range median of their small offset-corrected light curves. This can reject the upper and lower 5\% detections to avoid those outliers (i.e., the detections obviously deviated from the expected maximum/minimum of the folded light curve). Such outliers could be the detections contaminated by the nearby bright stars or the artifacts not filtered out from the light-curve extraction. These objects need follow-up observations to confirm their rotation periods. We will have more discussion of these cases in Section 4.2. For an overview of our data analysis procedure, we show the flow chart in Figure 3. 


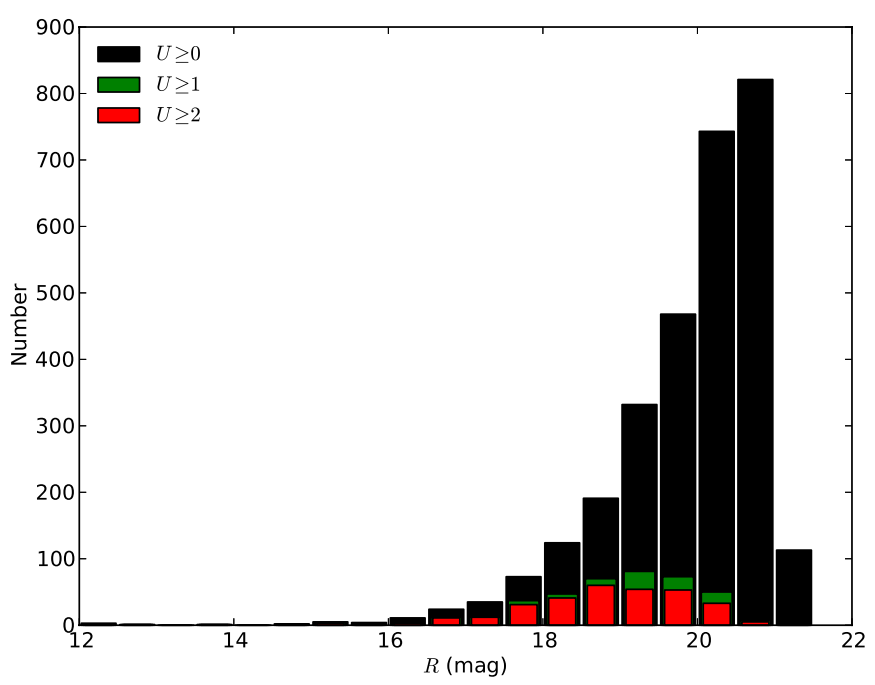

Figure 4. Distributions of magnitudes of the PTF-detected asteroids. Black, green, and red represent rotation periods of $U \geqslant 0, U \geqslant 1$, and $U \geqslant 2$, respectively.

(A color version of this figure is available in the online journal.)

\section{RESULTS}

\subsection{Detected Asteroids}

There were 2500 PTF-detected asteroids in a magnitude range of 1422 mag in this work (see Figure 4), and their distributions of the semimajor axes $(a)$, eccentricities $(e)$, inclinations $(i)$, and absolute magnitudes $\left(H_{R}\right)$ along with that of all known asteroids with $a<6 \mathrm{AU}$ are shown in Figure 5. The majority of the
PTF-detected asteroids were main belt asteroids, and the others were a few Hilda and Jovian Trojan asteroids and near-Earth objects. The PTF detection rate is higher for the inner main belt asteroids and fair to all eccentricities. Because we focused on the ecliptic plane, most PTF-detected asteroids concentrate on low inclinations.

The diameters of the PTF-detected asteroids were obtained from the preliminary results of WISE/NEOWISE (Grav et al. 2011; Mainzer et al. 2011; Masiero et al. 2011). While the WISE/NEOWISE diameters were not yet available, the diameters were estimated by the following equation:

$$
D=\frac{1130}{\sqrt{p_{R}}} 10^{-H_{R} / 5},
$$

where $D$ is the diameter in units of $\mathrm{km}, p_{R}$ is the geometric albedo in the $R$ band, and the conversion constant, 1130, is adopted from Jewitt et al. (2013). According to the semimajor axis ranges, we used three empirical values for geometric albedo, which are (1) $p=0.20$ for $a \leqslant 2.5 \mathrm{AU}$, (2) $p=0.08$ for $2.5<a \leqslant 2.8 \mathrm{AU}$, and (3) $p=0.04$ for $a>2.8$ AU (Tedesco et al. 2005). The plot of the semimajor axis versus diameter for the PTF-detected asteroids is given in Figure 6, which shows that the PTF was able to detect objects a few hundred meters in size in the inner main belt, kilometer-sized objects in the outer main belt, and objects $10 \mathrm{~km}$ in size for Hilda/Jovian Trojan asteroids.

\subsection{Derived Rotation Periods}

Among the 2500 PTF-detected asteroids, 313 objects had rotation periods with $U \geqslant 2$ (hereafter, the PTF-U2 asteroids). The information on the PTF-U2 asteroids is summarized in Table 2, and their folded light curves are presented in Figures 7-16.
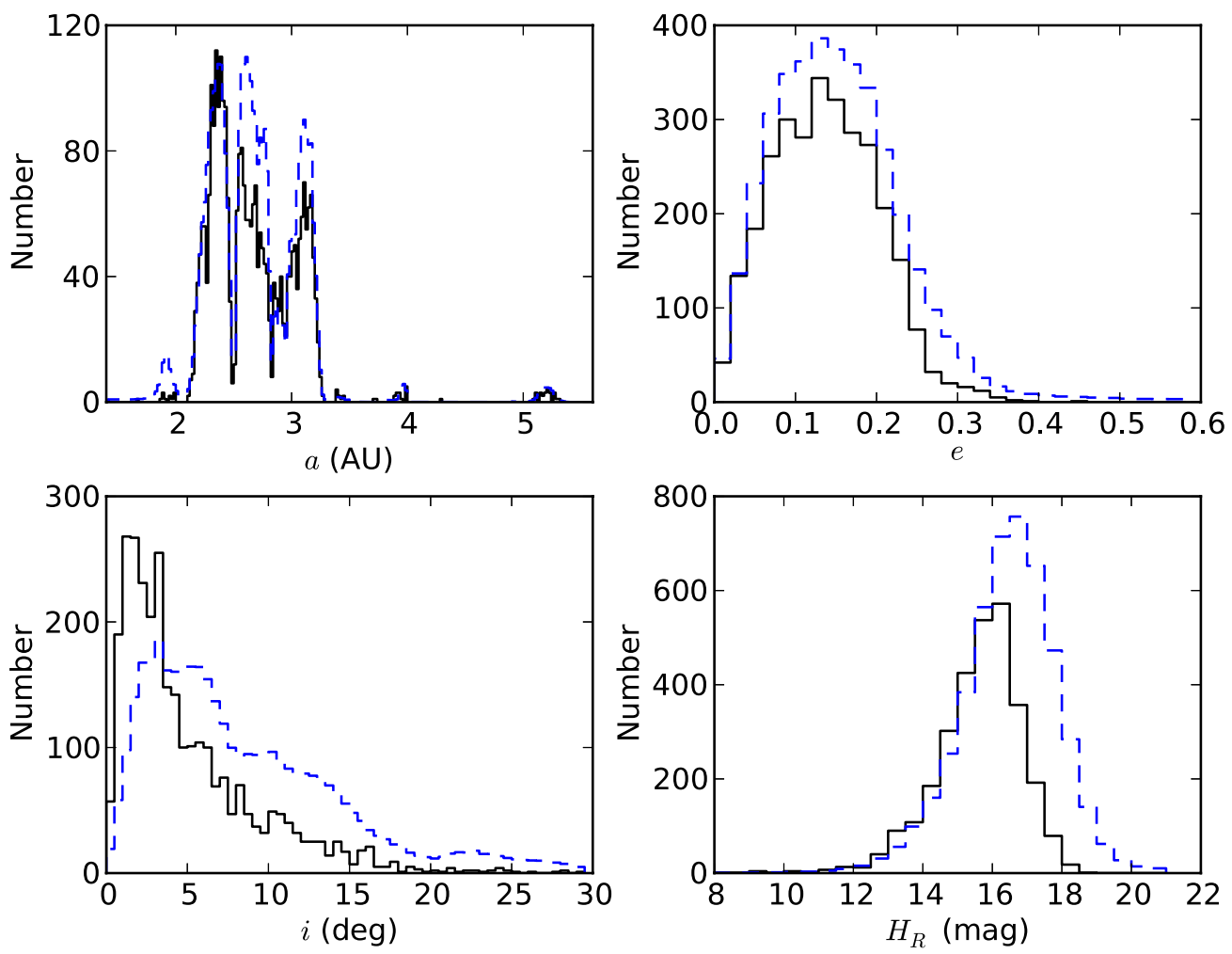

Figure 5. Distributions of orbital elements for the PTF-detected asteroids (solid line) along with those of known asteroids with $a<6$ AU in arbitrary normalization (dashed line). From the upper-left to the lower-right panels are the distributions for semimajor axes $(a, 0.02 \mathrm{AU}$ bins), eccentricities (e, 0.01 bins), inclinations ( $i, 0.5$ bins), and absolute magnitudes $\left(H_{R}, 0.3\right.$ mag bins).

(A color version of this figure is available in the online journal.) 
Table 2

Synodic Rotation Periods of 313 Asteroids with $U \geqslant 2$

\begin{tabular}{|c|c|c|c|c|c|c|c|c|c|c|c|c|c|c|c|c|c|c|}
\hline Obj ID & Designation & $a$ & $e$ & $i$ & $\Omega$ & $\omega$ & Epoch & $D$ & $\triangle$ & $r$ & $\alpha$ & $H$ & $n$ & $m$ & $\mathrm{PTF}_{R}$ & $\begin{array}{c}\text { Period } \\
(\mathrm{hr})\end{array}$ & $\triangle m$ & $U$ \\
\hline $00182^{a, b}$ & (182) Elsa & 2.42 & 0.19 & 2.00 & 107.2 & 310.2 & K13B4 & $44.0^{\mathrm{c}}$ & 2.18 & 1.32 & 16.50 & $9.07 \pm 0.33$ & 4 & 84 & $12.38 \pm 0.00$ & $15.97 \pm 0.31$ & 0.34 & \\
\hline $00300^{\mathrm{a}}$ & (300) Geraldina & 3.21 & 0.06 & 0.73 & 42.7 & 327.1 & K134I & $72.7^{\mathrm{c}}$ & 3.23 & 2.52 & 13.82 & $9.51 \pm 0.09$ & 4 & 57 & $14.85 \pm 0.00$ & $6.86 \pm 0.02$ & 0.16 & \\
\hline $00543^{\mathrm{a}}$ & (543) Charlotte & 3.06 & 0.16 & 8.48 & 295.0 & 109.8 & K134I & $45.6^{c}$ & 2.87 & 2.06 & 13.24 & $9.23 \pm 0.09$ & 4 & 61 & $13.85 \pm 0.00$ & $10.69 \pm 0.05$ & 0.20 & \\
\hline $00662^{a, b}$ & (662) Newtonia & 2.55 & 0.22 & 4.12 & 133.7 & 165.7 & K134I & $22.4^{\mathrm{c}}$ & 3.11 & 2.29 & 12.05 & $10.00 \pm 0.15$ & 4 & 47 & $15.06 \pm 0.00$ & $20.60 \pm 0.18$ & 0.42 & 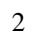 \\
\hline $00758^{a, b}$ & (758) Mancunia & 3.19 & 0.15 & 5.61 & 106.2 & 315.2 & K134I & $85.0^{\mathrm{c}}$ & 2.87 & 2.09 & 14.07 & $8.11 \pm 0.16$ & 4 & 62 & $12.85 \pm 0.00$ & $12.77 \pm 0.05$ & 0.26 & \\
\hline $01350^{\mathrm{a}}$ & (1350) Rosselia & 2.86 & 0.09 & 2.94 & 139.6 & 238.5 & K134I & $21.1^{\mathrm{c}}$ & 2.86 & 2.12 & 15.20 & $10.36 \pm 0.25$ & 4 & 59 & $14.99 \pm 0.00$ & $8.16 \pm 0.01$ & 0.52 & \\
\hline $01633^{\mathrm{a}}$ & (1633) Chimay & 3.19 & 0.12 & 2.68 & 114.1 & 65.0 & K134I & $37.7^{\mathrm{c}}$ & 2.96 & 2.16 & 13.19 & $10.36 \pm 0.17$ & 4 & 55 & $15.21 \pm 0.00$ & $6.58 \pm 0.01$ & 0.34 & \\
\hline 01778 & (1778) Alfven & 3.15 & 0.13 & 2.47 & 106.2 & 135.8 & K134I & $20.6^{\mathrm{c}}$ & 3.33 & 2.51 & 11.15 & $11.59 \pm 0.15$ & 4 & 55 & $16.88 \pm 0.01$ & $4.82 \pm 0.00$ & 0.37 & \\
\hline 01913 & (1913) Sekanina & 2.88 & 0.08 & 1.57 & 358.5 & 35.2 & K134I & $13.4^{\mathrm{c}}$ & 2.86 & 2.01 & 12.35 & $11.64 \pm 0.15$ & 4 & 56 & $16.16 \pm 0.01$ & $13.97 \pm 0.06$ & 0.27 & \\
\hline $01955^{\mathrm{a}}$ & (1955) McMath & 2.85 & 0.07 & 1.01 & 258.2 & 154.7 & K134I & $9.8^{\mathrm{c}}$ & 2.75 & 2.05 & 16.84 & $11.97 \pm 0.15$ & 4 & 59 & $16.64 \pm 0.01$ & $5.57 \pm 0.00$ & 0.39 & \\
\hline
\end{tabular}

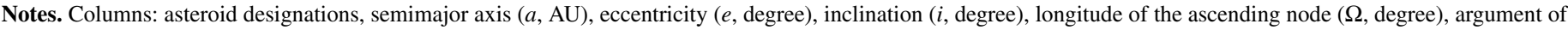

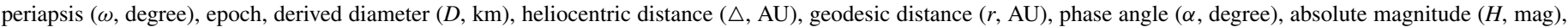

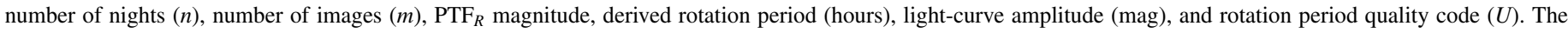
amplitudes of the objects of partial light-curve coverage and single minimum light curve should be treated as lower limits.

a The asteroids have published rotation periods.

b The objects of partial light-curve coverage.

c The WISE/NEOWISE diameter.

d The objects show a single minimum light curve.

(This table is available in its entirety in a machine-readable form in the online journal. A portion is shown here for guidance regarding its form and content.)

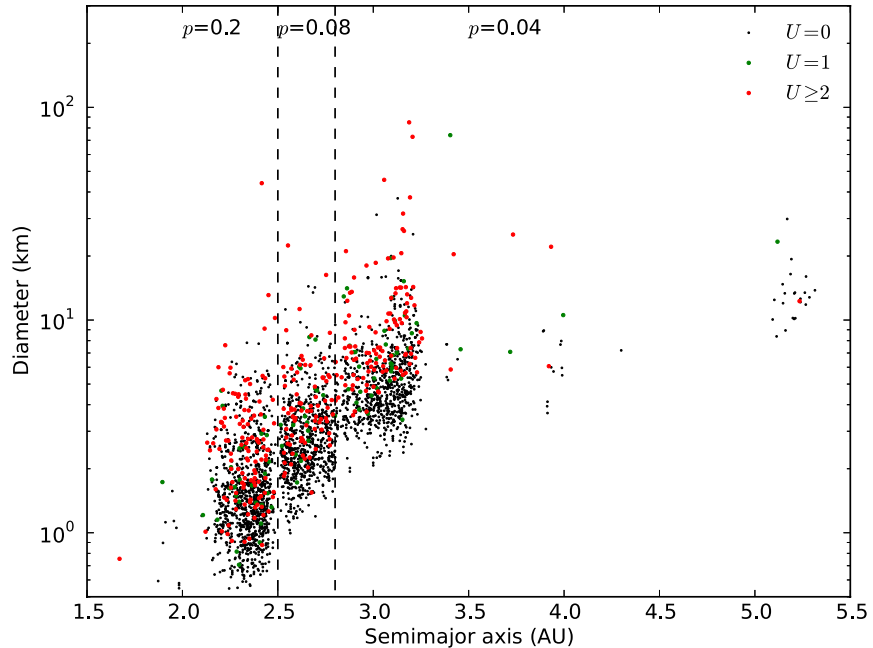

Figure 6. Plot of the diameter vs. semimajor axis for the PTF-detected objects. The red, gray, and black filled circles represent rotation periods of $U=0, U=$ 1 , and $U \leqslant 2$, respectively. The dashed lines show the semimajor axis ranges for different empirical values of geometric albedo $(p)$.

(A color version of this figure is available in the online journal.)

Eighteen PTF-U2 asteroids have published rotation periods in the Asteroid Light Curve Database (LCDB; Warner et al. 2009), ${ }^{10}$ and 16 of them show good agreements on their rotation periods, which indicated that our results were highly reliable. The possible reasons that the rotation periods of the other two asteroids, (182) Elsa and (16541) 1991 PW18, showed discrepancies between this work and the LCDB are discussed below.

Compared to the published rotation period of $>9 \mathrm{hr}$ with $U=$ 1 for 1991 PW18 (Warner et al. 2009), ours is $7.0 \mathrm{hr}$ with $U=2$, which showed a clear folded light curve; therefore, we believe our result to be more accurate.

The rotation period of (182) Elsa, a relatively large asteroid ( $\sim 40 \mathrm{~km}$ ), had been carefully studied by Pilcher et al. (2009)

\footnotetext{
10 http://www.minorplanet.info/lightcurvedatabase.html
}

with an intensively observed light curve. They gave a rotation period of $80.088 \mathrm{hr}$ and a clear folded light curve that showed similar primary and secondary minimums. Because our data set only covered part of its rotation period, the fitting procedure misidentifies a rotation period of $15.97 \mathrm{hr}$ (i.e., $\sim 1 / 5$ of the published result) and gives a clear folded light curve. As per the criteria described in Section 3.4, we still assigned $U=2$ to our rotation period.

From the case of (182) Elsa, we notice that when a folded light curve only covered part of a rotation period, our fitting procedure could possibly lead us to an inaccurate rotation period. In 312 PTF-U2 asteroids, we found 20 objects facing this situation, and five more showed folded light curves with a single minimum. Because these 25 objects possibly had relatively large uncertainties in their rotation periods, we exclude them in the following statistical analysis. The folded light curves of these 25 objects are presented in Figures 15 and 16, respectively.

There were 49 PTF-detected asteroids with $R<18$ mag and more than 20 detections that do not have rotation period determinations (see Table 3). Because the small offset corrections for most PTF-U2 asteroids (i.e., $>90 \%$ ) are of $R<0.1 \mathrm{mag}$ and the relative photometry accuracy of $R=18 \mathrm{mag}$ is $\sim 0.05 \mathrm{mag}$, it was unlikely to detect a light-curve variation of $R<0.1 \mathrm{mag}$ in our analysis, and this has also been seen in Figure 18. Among 49 objects, only five belonged to this case. The other 44 objects with light-curve variations of $R>0.1 \mathrm{mag}$ should have had a rotation period determination. However, most of these objects showed segmented light curves with a long trend over our four-night observation time span (i.e., the light-curve coverage was very limited). This indicates that these objects might have relatively long rotation periods (i.e., several days) and would have less chance to have rotation period determinations from our four-night observation. In addition, 18 out of these 44 objects had diameters of $>10 \mathrm{~km}$ and six of them (i.e., $~ 33 \%$ ) have light-curve variations of $R>0.3 \mathrm{mag}$. Compared to the remaining 26 objects, in which 10 objects $(\sim 38 \%)$ have light-curve variations of $R>0.3 \mathrm{mag}$, both groups showed similar fractions for light-curve variation. However, this result was based on the lower limit of the light-curve amplitude (i.e., we only had 

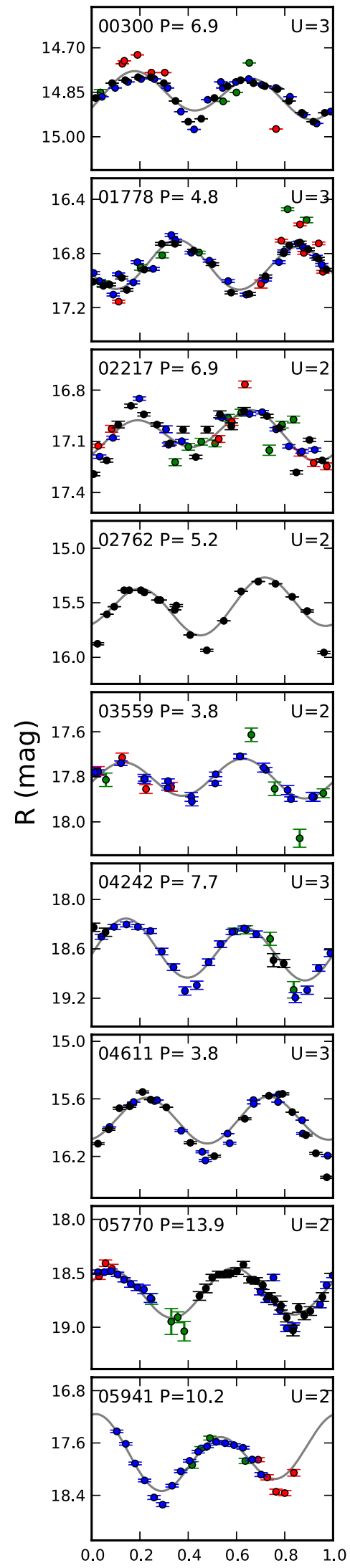
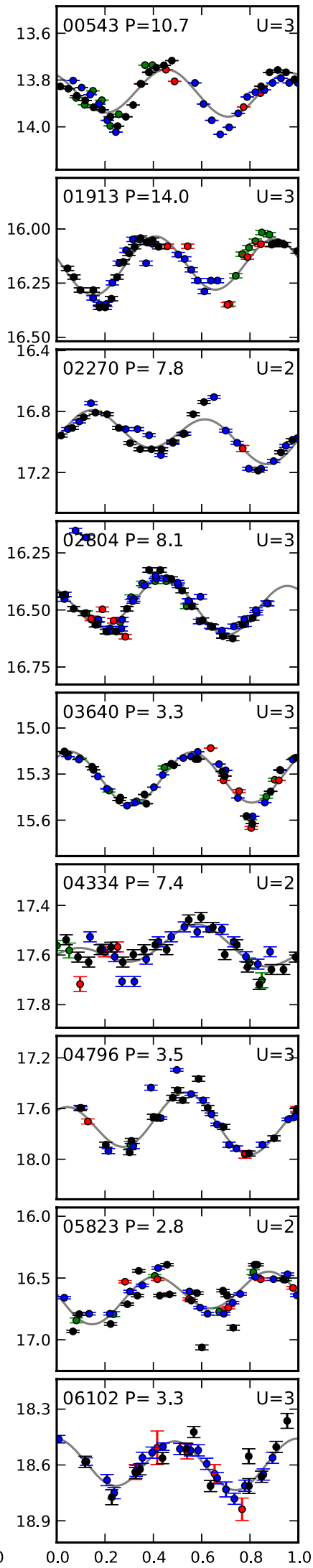
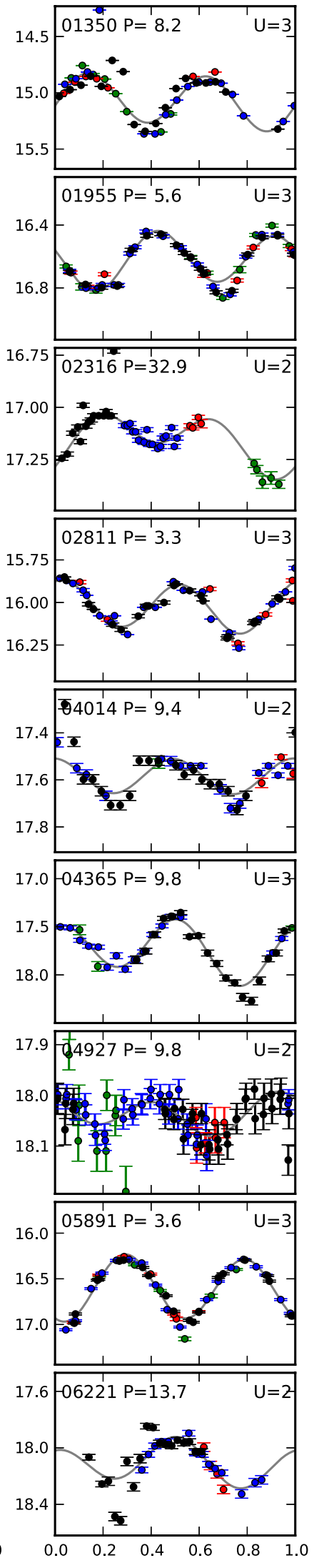

Phase
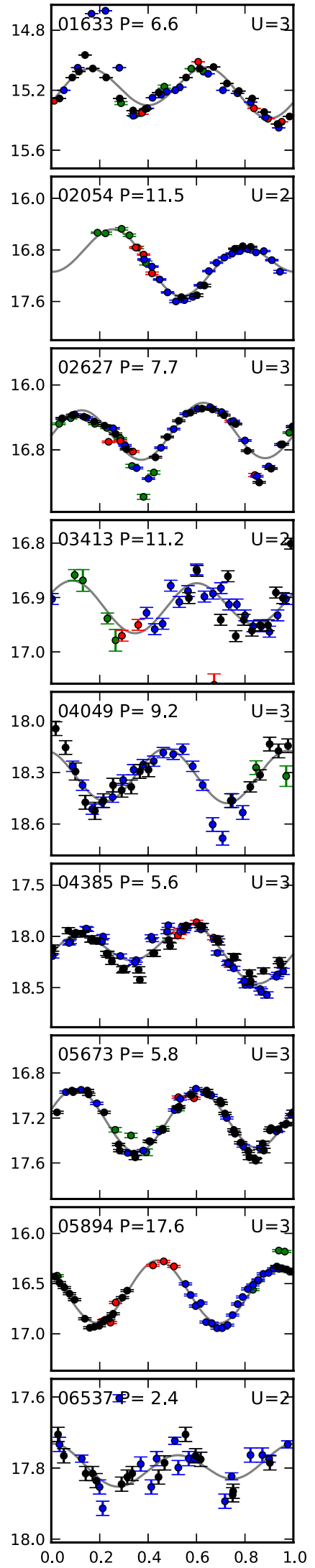

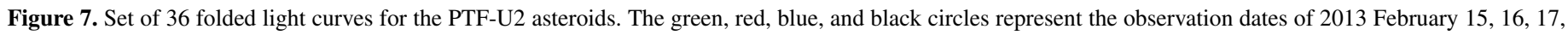
and 18, respectively. The designation, rotation period in hours $(P)$, and the quality code $(U)$ of each object are shown on each plot.

(A color version of this figure is available in the online journal.) 

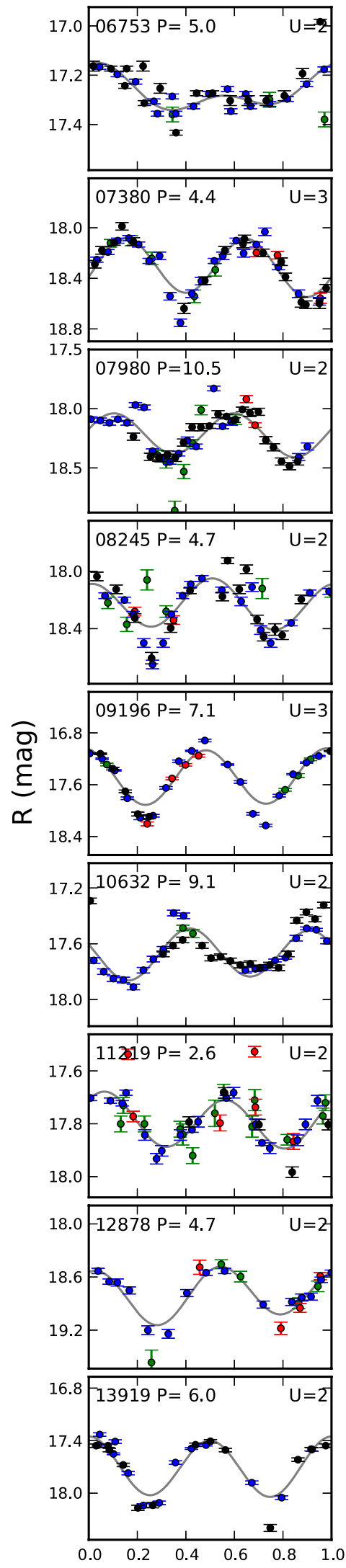
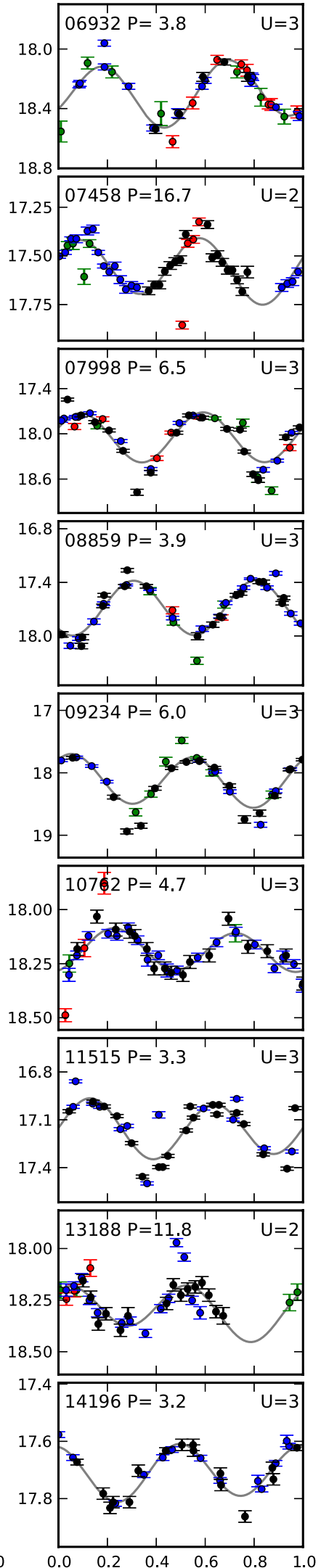
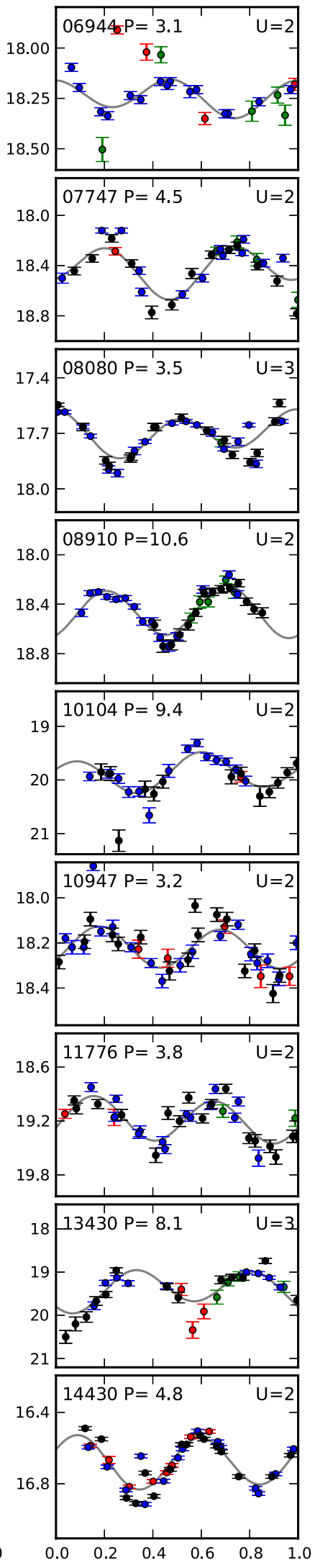

Phase

Figure 8. Same as Figure 7 for 36 more PTF-U2 asteroids.

(A color version of this figure is available in the online journal.)
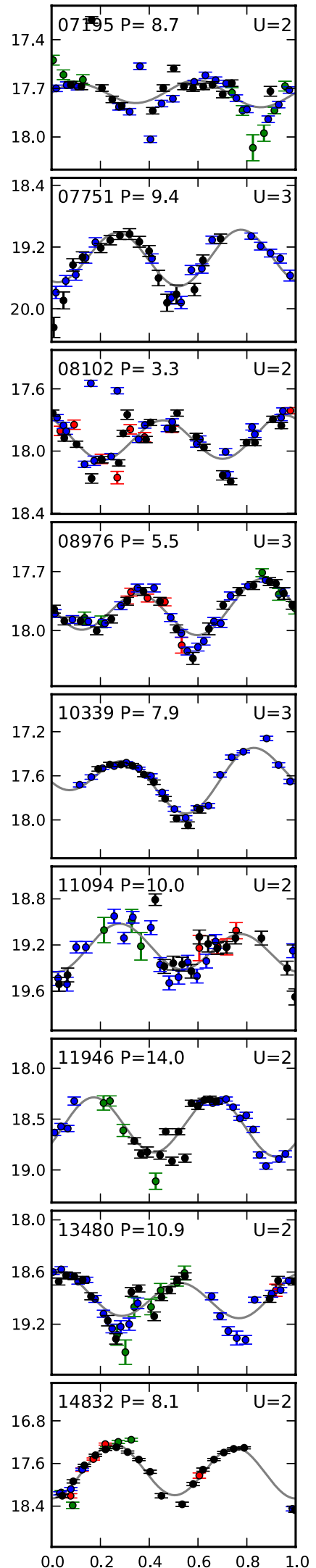


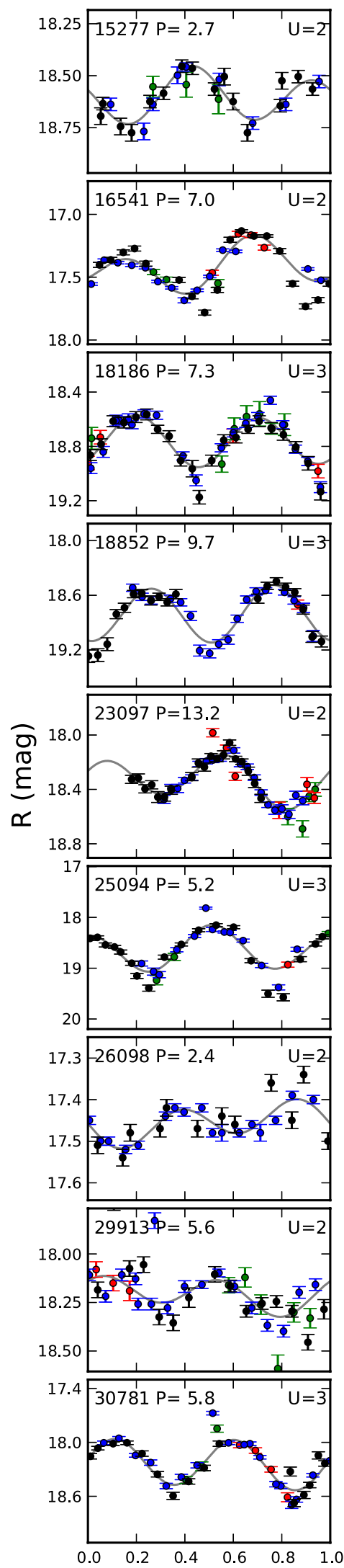

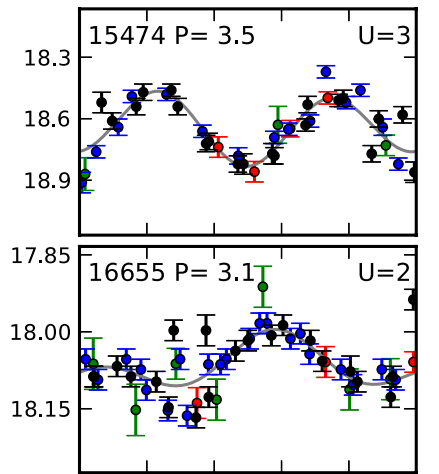
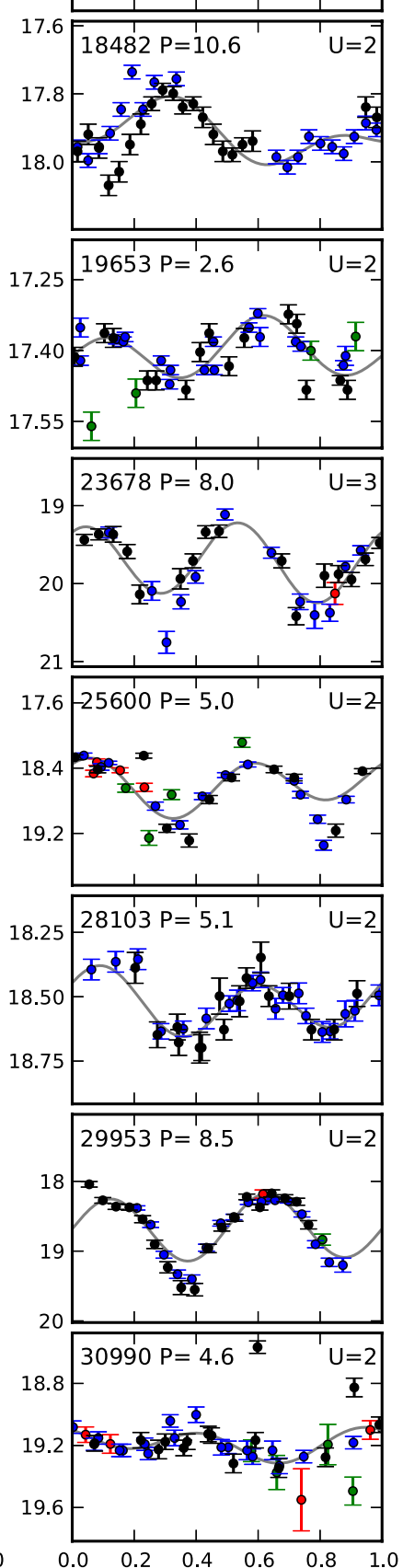
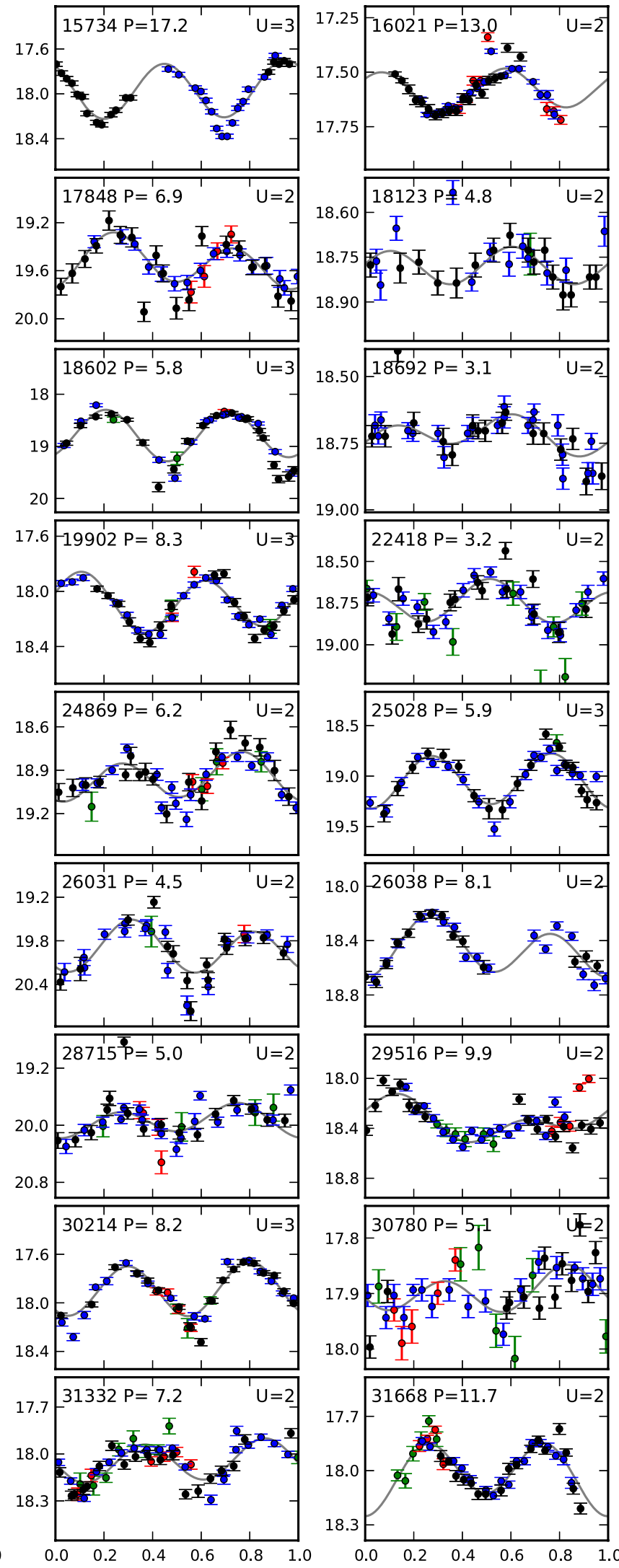

\section{Phase}

Figure 9. Same as Figure 7 for 36 more PTF-U2 asteroids.

(A color version of this figure is available in the online journal.) 

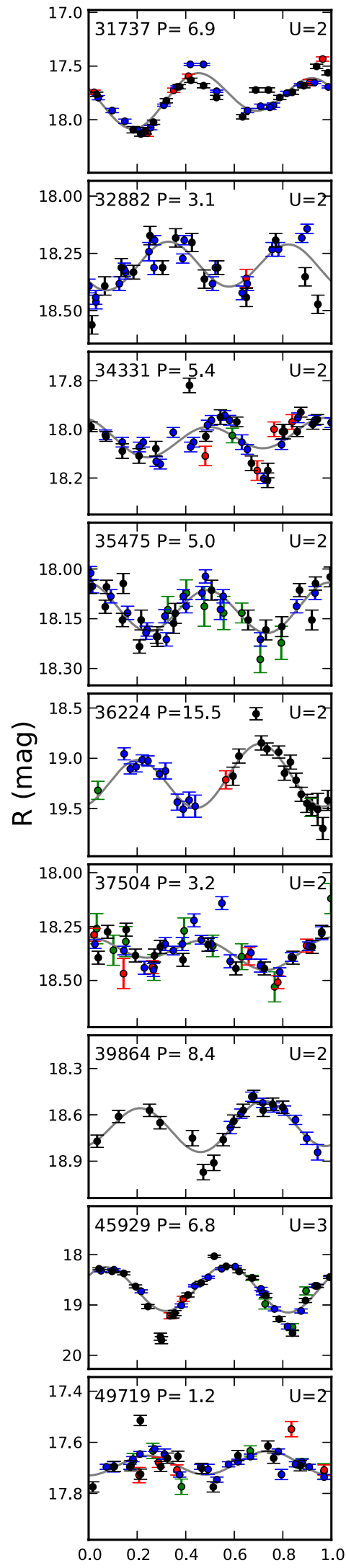
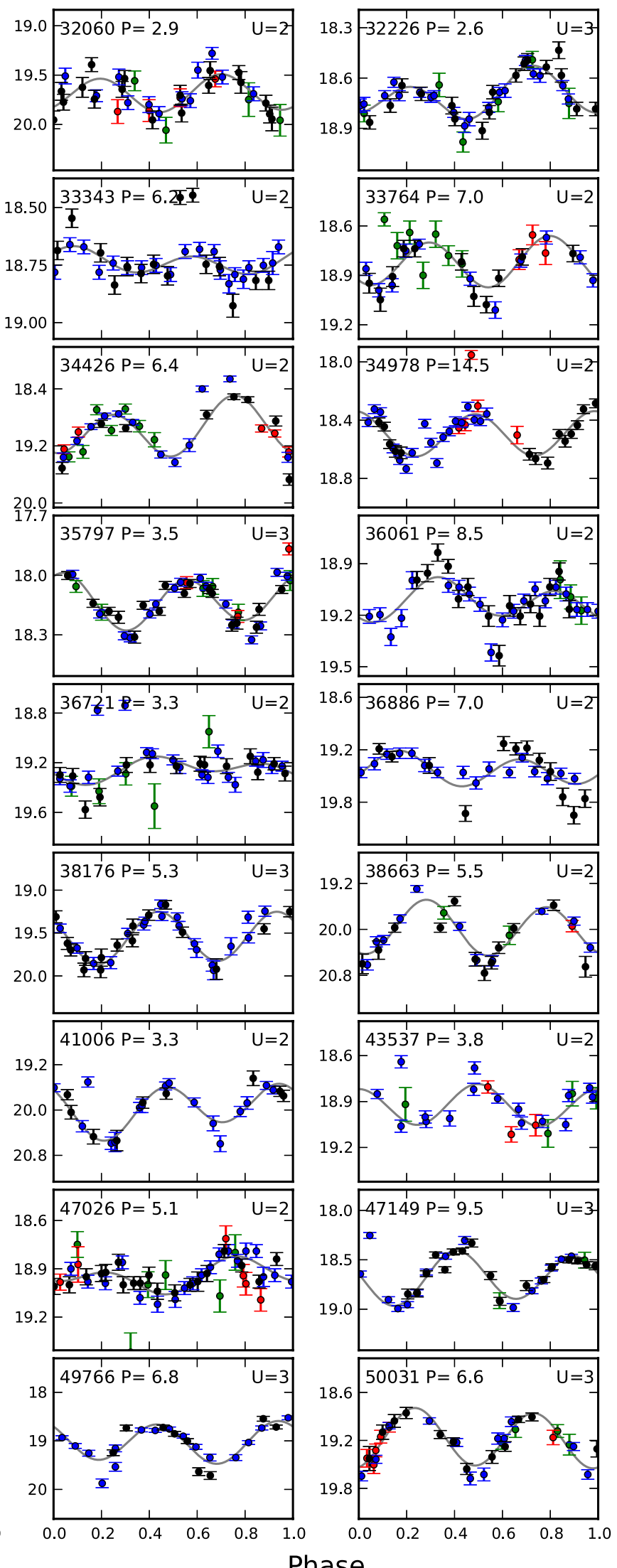

Phase

Figure 10. Same as Figure 7 for 36 more PTF-U2 asteroids.

(A color version of this figure is available in the online journal.)
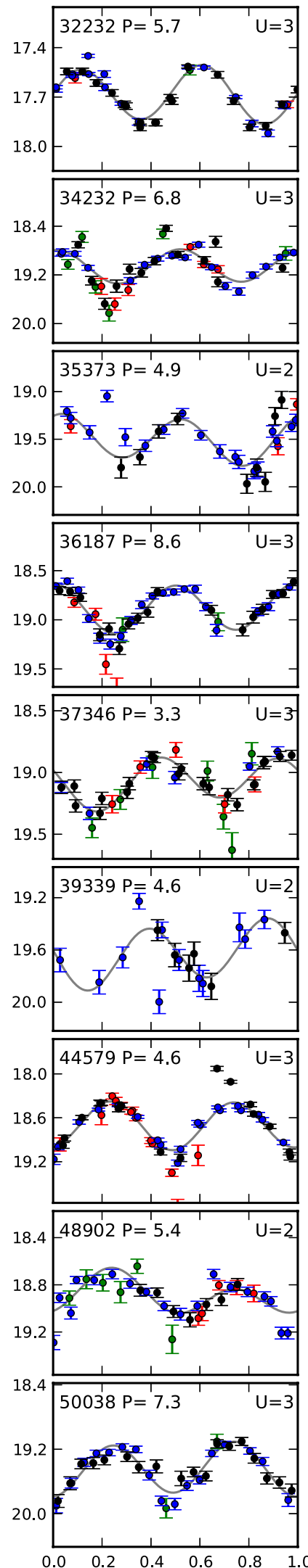


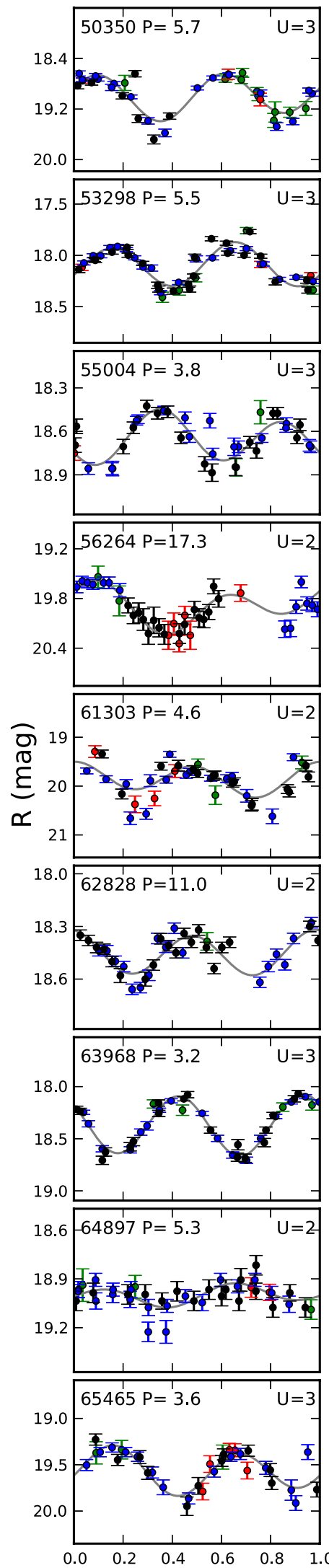

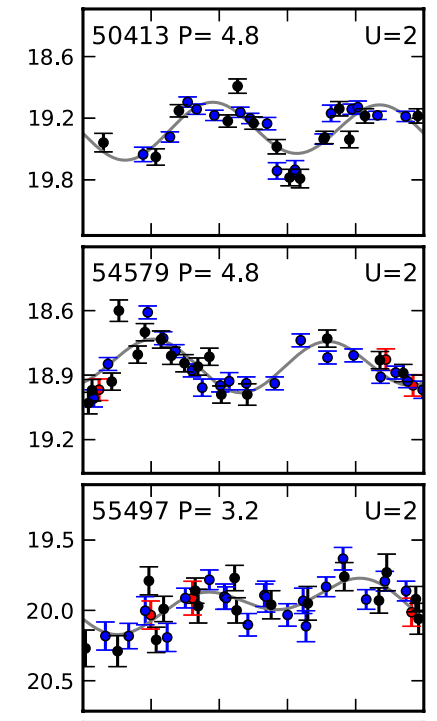
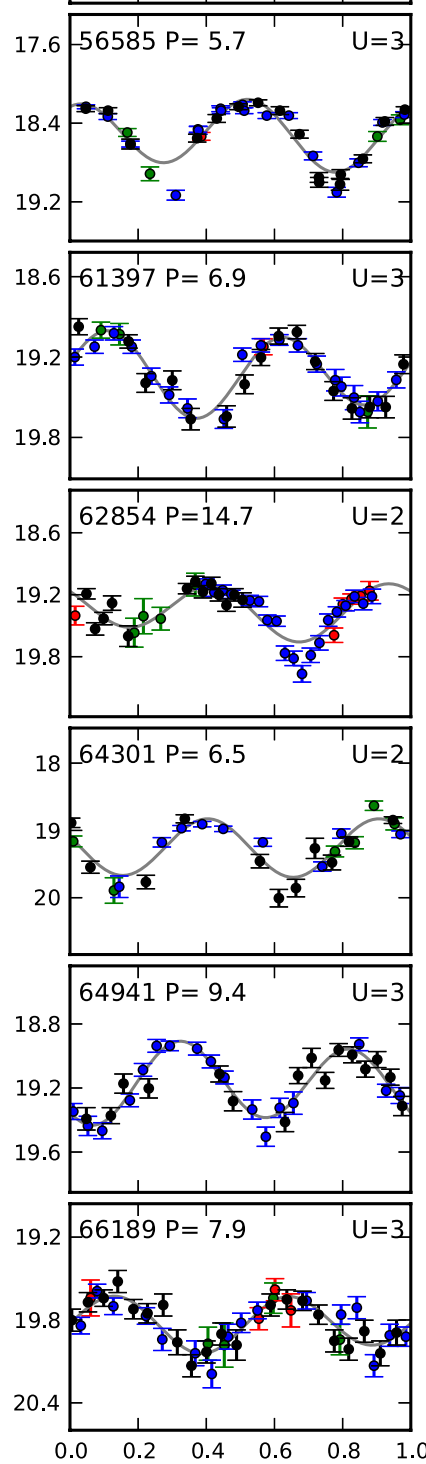
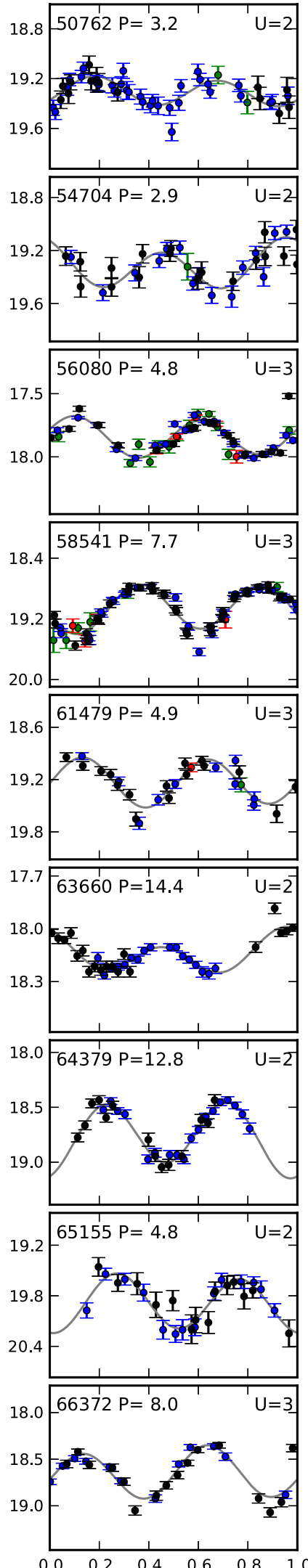

Phase

Figure 11. Same as Figure 7 for 36 more PTF-U2 asteroids.

(A color version of this figure is available in the online journal.)
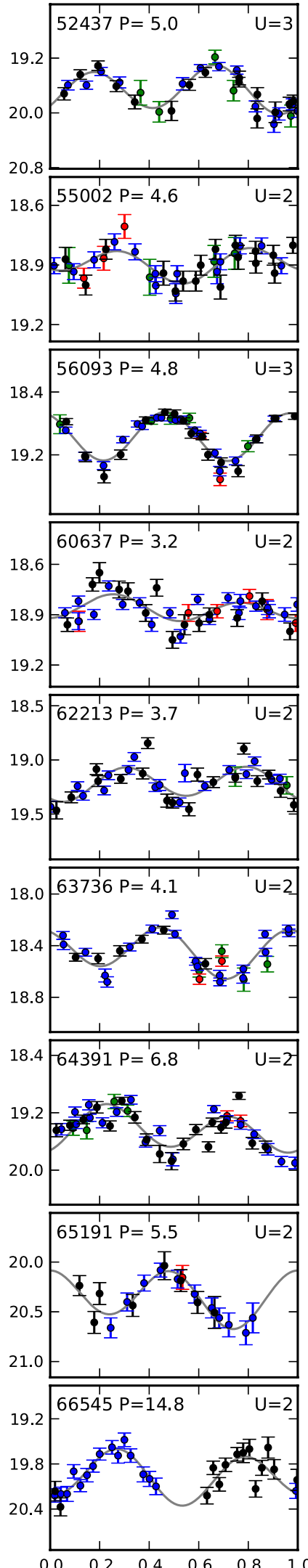


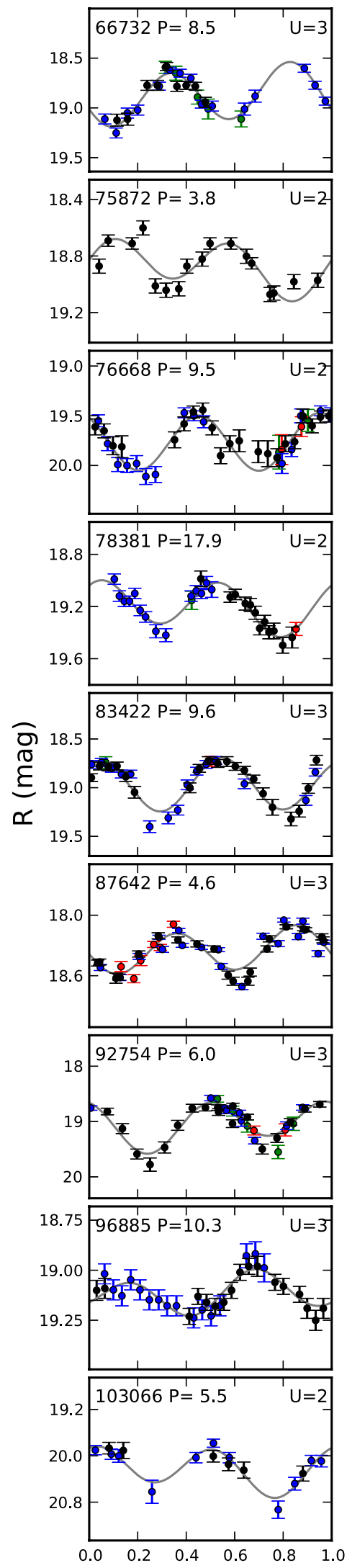

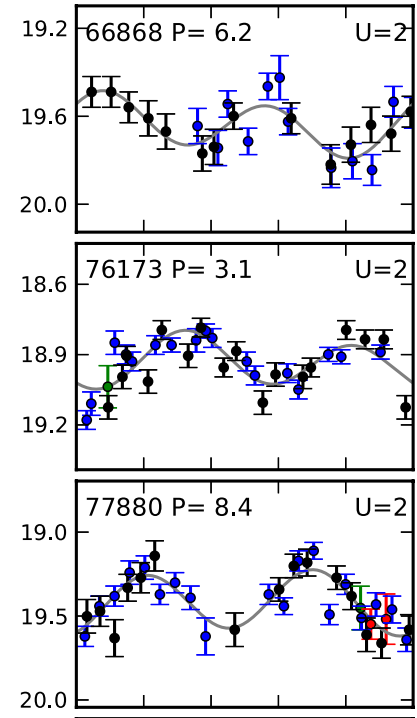
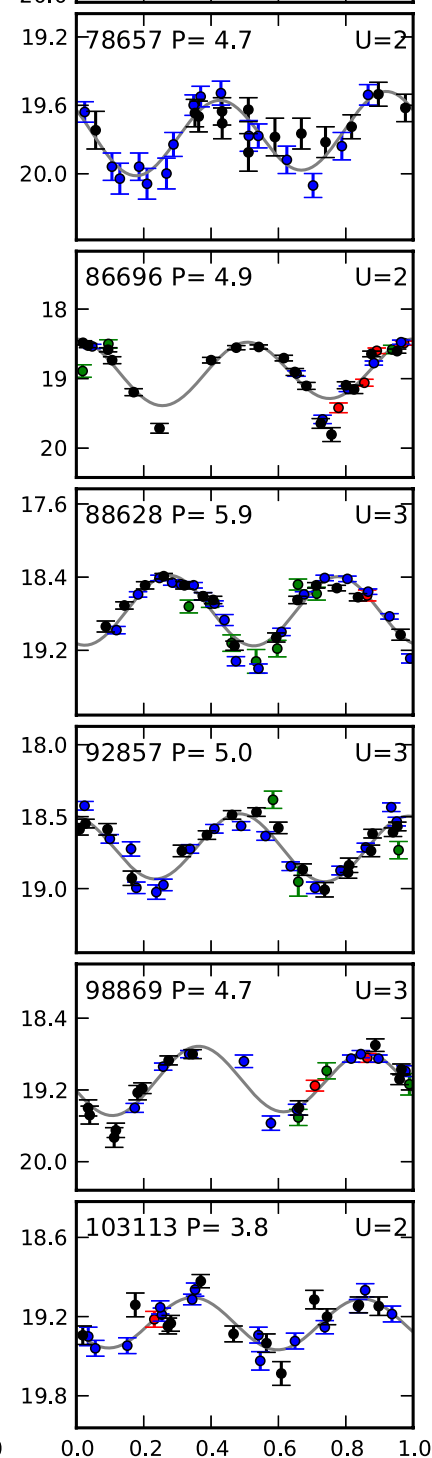
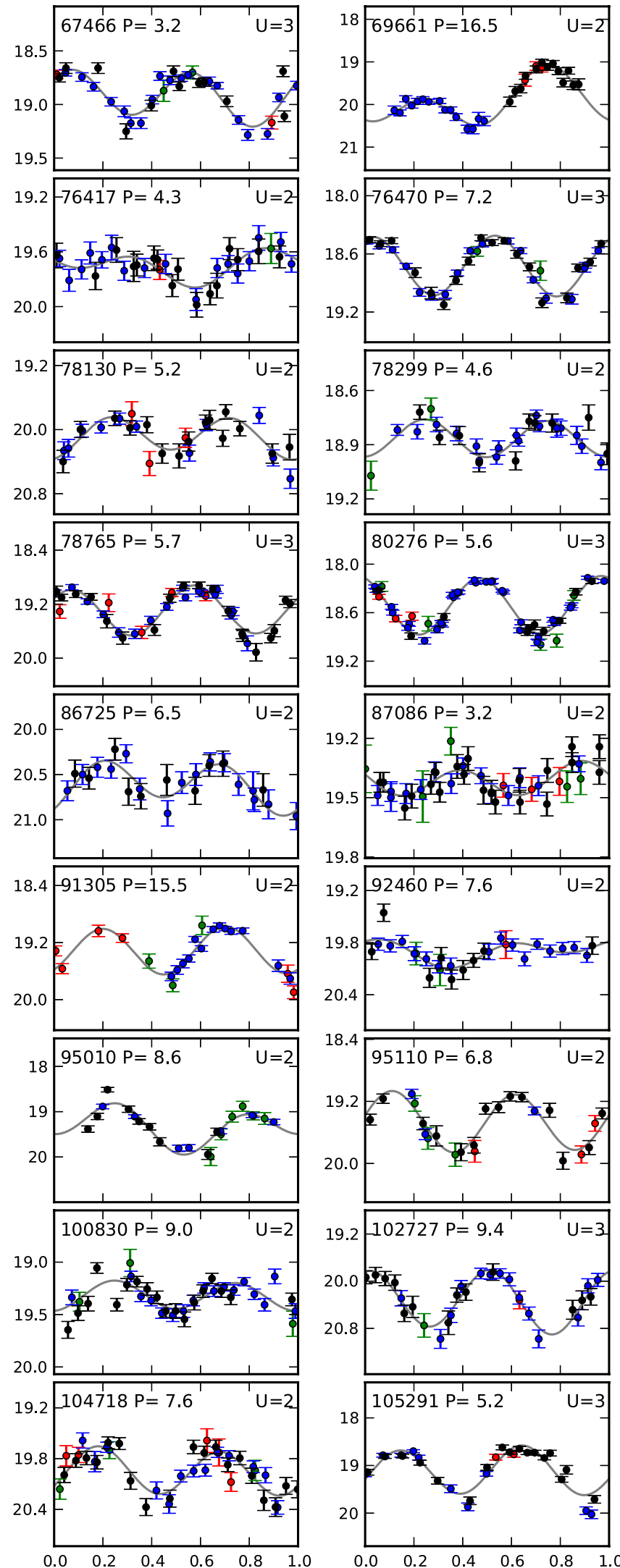

\section{Phase}

Figure 12. Same as Figure 7 for 36 more PTF-U2 asteroids.

(A color version of this figure is available in the online journal.) 


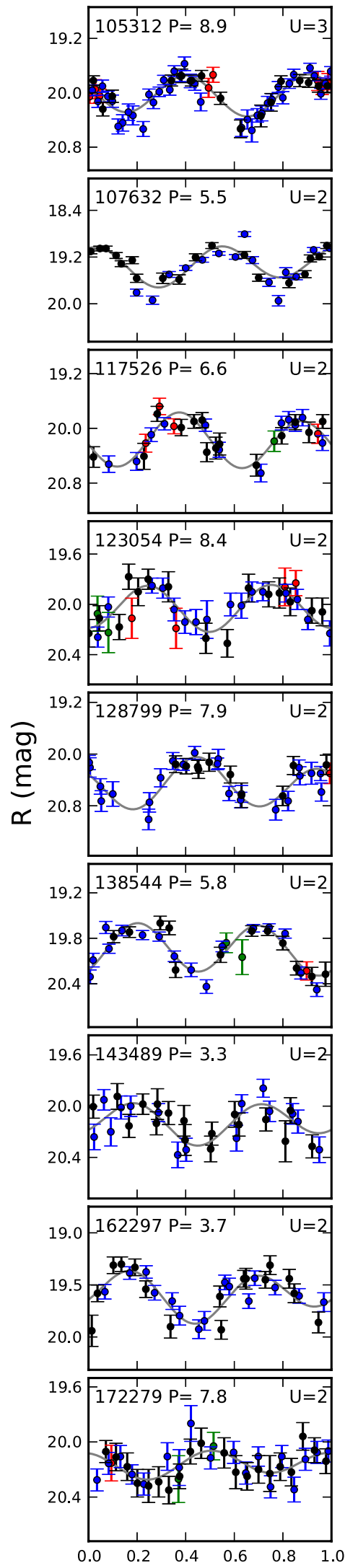

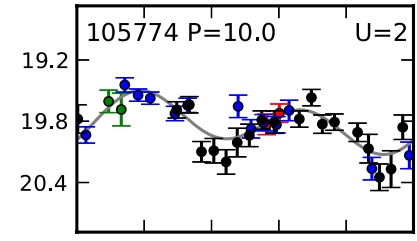
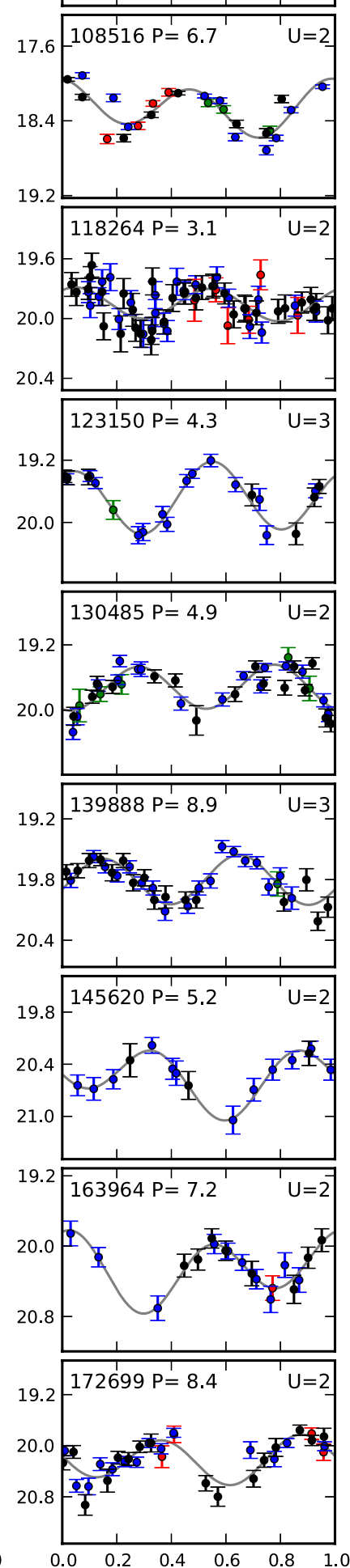
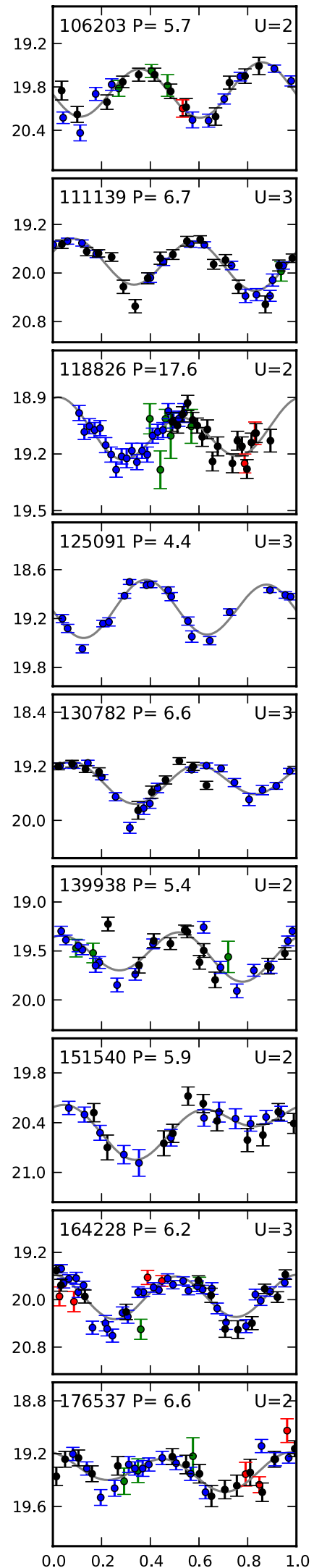

Phase

Figure 13. Same as Figure 7 for 36 more PTF-U2 asteroids.

(A color version of this figure is available in the online journal.)
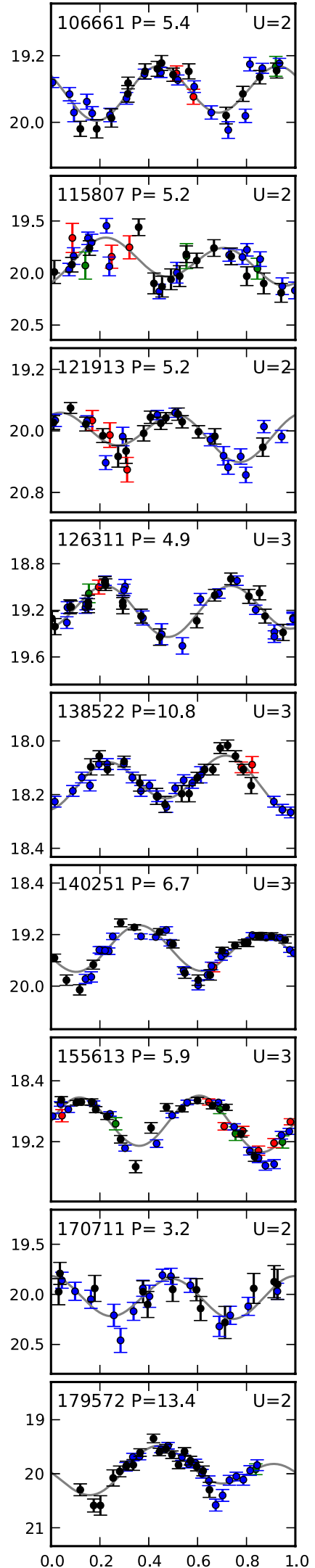


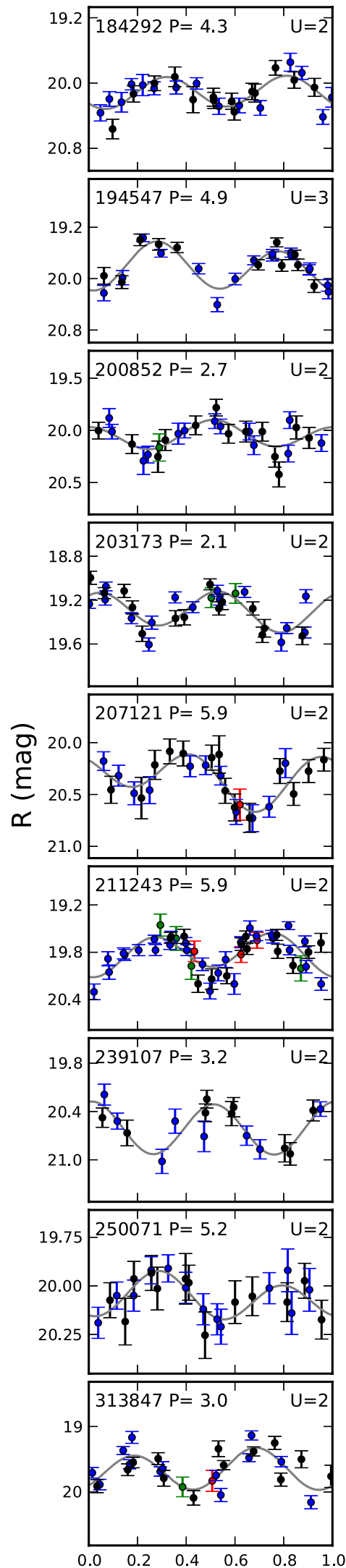

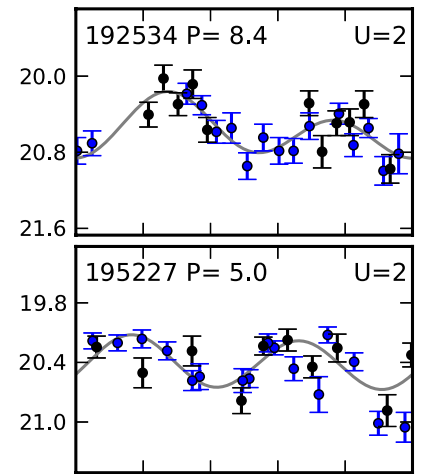
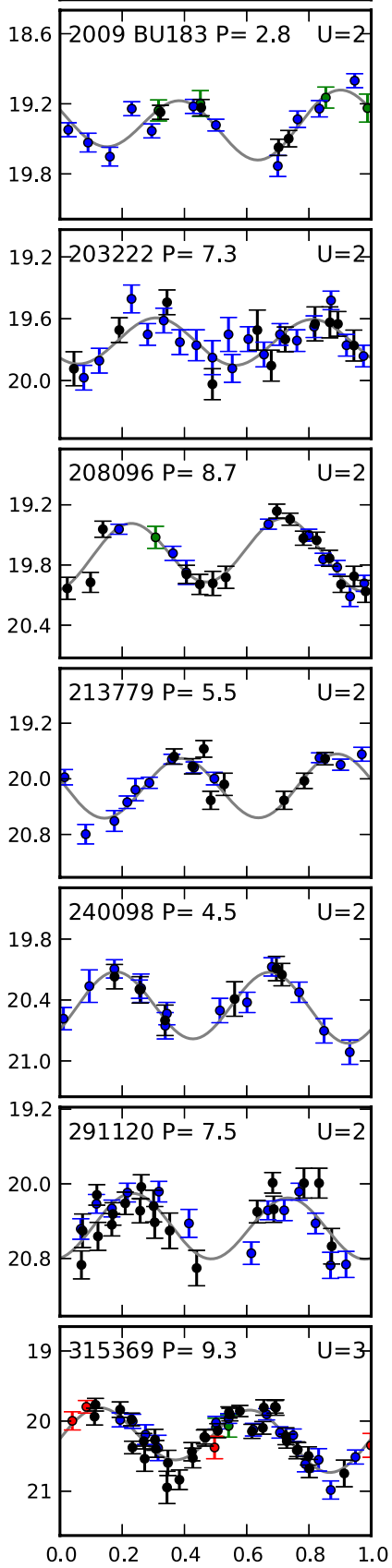
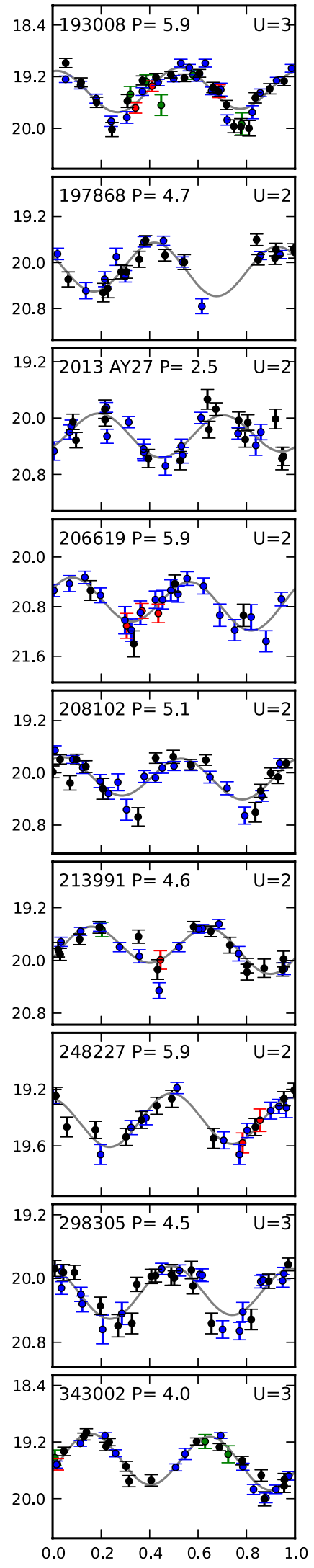

Phase

Figure 14. Same as Figure 7 for 36 more PTF-U2 asteroids

(A color version of this figure is available in the online journal.)
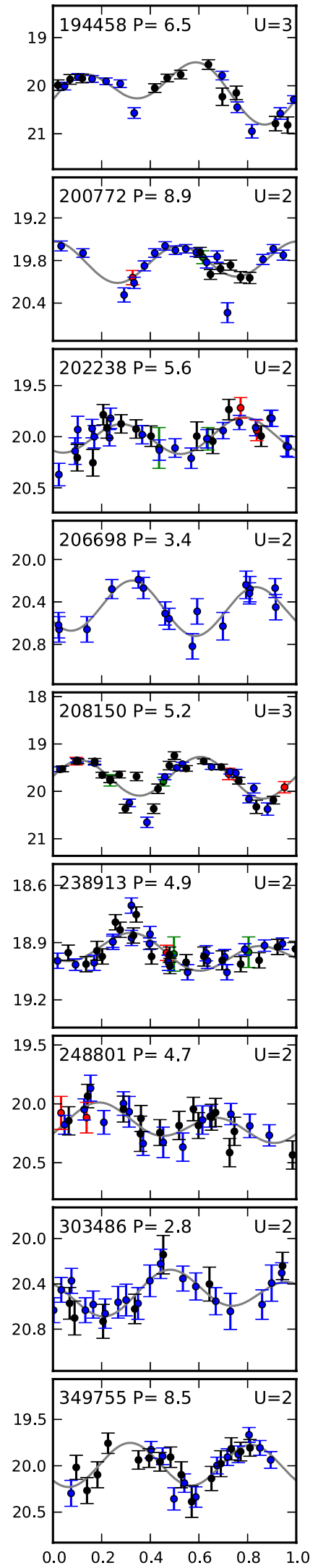

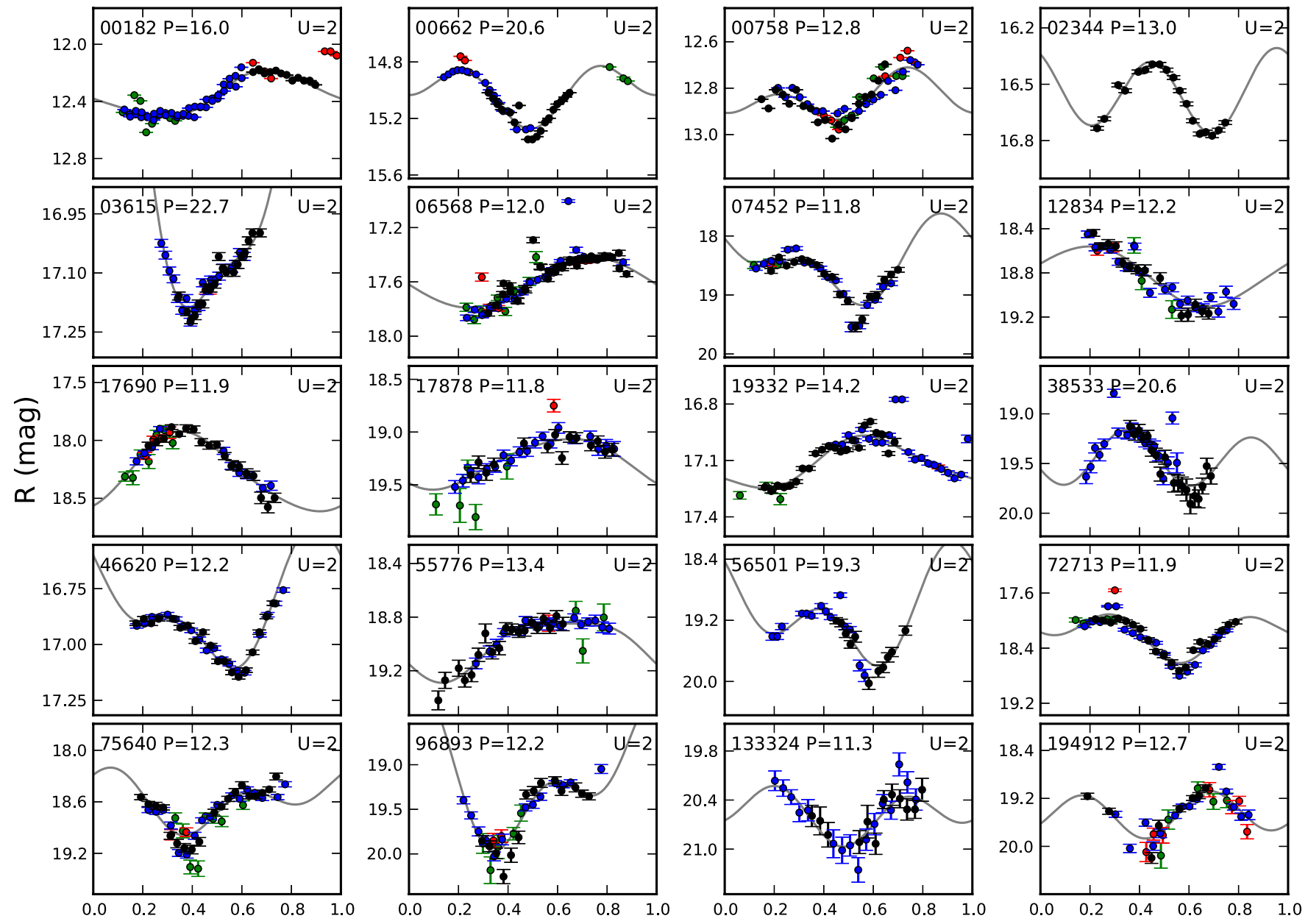

Phase

Figure 15. Same as Figure 7 for 20 more PTF-U2 asteroids, whose folded light curves only cover part of a rotation period. (7452) Izabelyuria and (75640) 2000 AE55 show binary asteroid features.

(A color version of this figure is available in the online journal.)
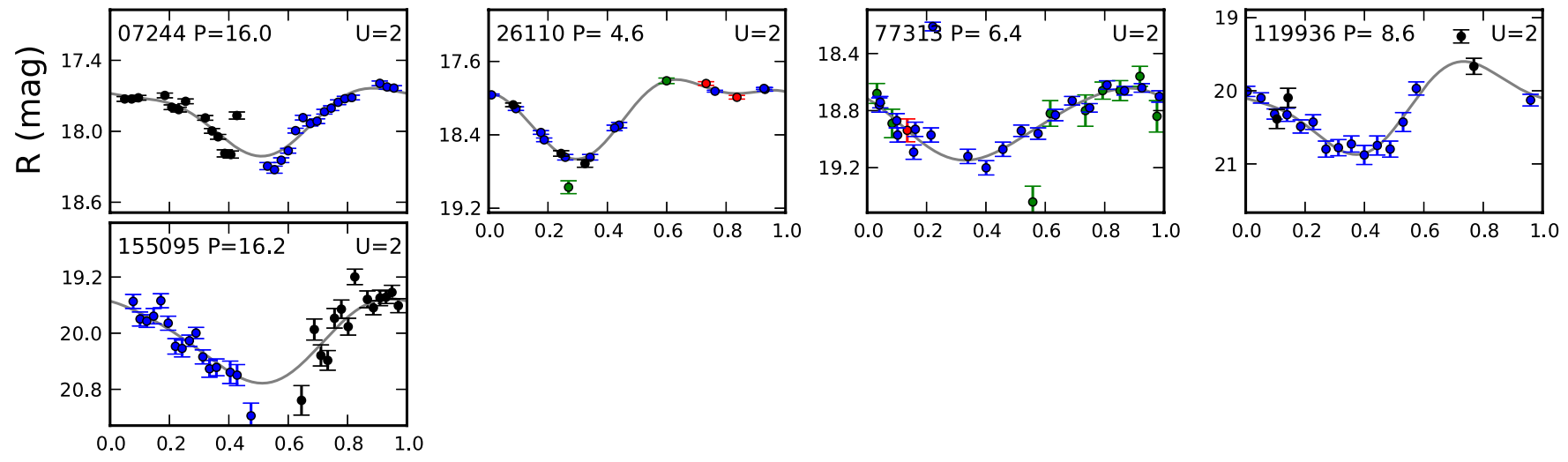

\section{Phase}

Figure 16. Same as Figure 7 for five more PTF-U2 asteroids, whose folded light curves show a single minimum.

(A color version of this figure is available in the online journal.)

partial light-curve coverage) and should be verified when their full light curves are available.

\subsection{Statistical Analysis}

The asteroid spin-rate limit is one of the particularly interesting subjects. Figure 17 shows the plot of the diameter versus rotation period for the PTF-U2 asteroids and the objects adopted from the LCDB with $U \geqslant 2$. The PTF-U2 asteroids occupy the dense region of the plot. Because our observation was insensitive to detecting a long rotation period, the PTF-U2 asteroids showed a lack of slow rotators. The "spin barrier" at $\sim 2.2 \mathrm{hr}$ can clearly be seen for asteroids with diameters larger than a few hundred meters, which indicated the spin-rate limit for gravitationally 
Table 3

The 49 Bright PTF-detected Asteroids with $U \leqslant 1$

\begin{tabular}{|c|c|c|c|c|c|c|c|c|c|c|c|c|c|c|c|c|c|c|}
\hline Obj ID & Designation & $a$ & $e$ & $i$ & $\Omega$ & $\omega$ & Epoch & $D$ & $\triangle$ & $r$ & $\alpha$ & $H$ & $n$ & $m$ & $\mathrm{PTF}_{R}$ & $\begin{array}{c}\text { Period } \\
\text { (hr) }\end{array}$ & $\Delta m$ & $U$ \\
\hline 01004 & (1004) Belopolskya & 3.40 & 0.09 & 2.98 & 153.6 & 216.3 & K134I & 74.0 & 3.44 & 2.71 & 12.56 & $9.41 \pm 0.08$ & 4 & 63 & $15.00 \pm 0.00$ & $9.06 \pm 0.03$ & 0.12 & 1 \\
\hline 01780 & (1780) Kippes & 3.02 & 0.05 & 9.00 & 291.0 & 339.8 & K134I & $31.3^{\mathrm{a}}$ & 3.17 & 2.54 & 15.39 & $10.15 \pm 0.06$ & 4 & 59 & $15.53 \pm 0.01$ & & 0.11 & \\
\hline 01986 & (1986) Plaut & 3.09 & 0.20 & 2.21 & 146.6 & 230.8 & K134I & $19.6^{\mathrm{a}}$ & 3.02 & 2.28 & 14.37 & $11.82 \pm 0.07$ & 3 & 31 & $16.83 \pm 0.01$ & $13.62 \pm 0.54$ & 0.16 & 1 \\
\hline 02264 & (2264) Sabrina & 3.13 & 0.17 & 0.16 & 240.3 & 79.1 & K134I & $37.3^{\mathrm{a}}$ & 3.60 & 2.85 & 11.62 & $10.50 \pm 0.10$ & 4 & 50 & $16.30 \pm 0.01$ & & 0.19 & \\
\hline 02273 & (2273) Yarilo & 2.45 & 0.17 & 0.39 & 66.1 & 41.5 & K134I & $5.6^{\mathrm{a}}$ & 2.06 & 1.21 & 18.71 & $13.50 \pm 0.07$ & 4 & 65 & $16.46 \pm 0.01$ & & 0.10 & \\
\hline 02668 & (2668) Tataria & 2.32 & 0.08 & 3.16 & 298.2 & 62.9 & K134I & $5.4^{\mathrm{a}}$ & 2.37 & 1.69 & 20.82 & $13.22 \pm 0.25$ & 4 & 50 & $17.24 \pm 0.02$ & & 0.53 & \\
\hline 03187 & (3187) Dalian & 2.28 & 0.06 & 2.76 & 312.4 & 105.1 & K134I & $6.2^{\mathrm{a}}$ & 2.21 & 1.44 & 19.78 & $13.02 \pm 0.11$ & 4 & 54 & $16.53 \pm 0.01$ & & 0.22 & \\
\hline 03462 & (3462) Zhouguangzhao & 2.45 & 0.21 & 5.80 & 113.1 & 254.9 & K134I & 5.5 & 2.46 & 1.77 & 19.41 & $13.32 \pm 0.40$ & 4 & 42 & $17.42 \pm 0.02$ & & 0.75 & \\
\hline 03935 & (3935) Toatenmongakkai & 2.54 & 0.23 & 8.75 & 300.5 & 104.4 & K134I & $11.2^{\mathrm{a}}$ & 2.24 & 1.49 & 20.26 & $11.78 \pm 0.42$ & 4 & 49 & $15.43 \pm 0.01$ & & 1.08 & \\
\hline 04890 & (4890) Shikanosima & 2.20 & 0.15 & 3.66 & 100.3 & 185.5 & K134I & $4.7^{\mathrm{a}}$ & 2.52 & 1.70 & 15.46 & $13.52 \pm 0.11$ & 4 & 45 & $17.52 \pm 0.01$ & & 0.21 & \\
\hline 04929 & (4929) Yamatai & 2.21 & 0.06 & 2.49 & 127.5 & 89.9 & K134I & $4.0^{\mathrm{a}}$ & 2.22 & 1.42 & 19.08 & $14.04 \pm 0.07$ & 2 & 36 & $17.50 \pm 0.02$ & $3.62 \pm 0.06$ & 0.14 & 1 \\
\hline 05005 & (5005) Kegler & 2.25 & 0.17 & 1.31 & 320.7 & 108.7 & K134I & $3.5^{\mathrm{a}}$ & 1.98 & 1.18 & 21.69 & $14.61 \pm 0.10$ & 4 & 35 & $17.52 \pm 0.02$ & & 0.31 & \\
\hline 05045 & (5045) Hoyin & 3.13 & 0.19 & 2.57 & 130.7 & 262.5 & K134I & 17.1 & 3.00 & 2.16 & 11.96 & $12.60 \pm 1.30$ & 4 & 40 & $17.60 \pm 0.02$ & & 0.47 & \\
\hline 05199 & (5199) Dortmund & 2.62 & 0.18 & 12.27 & 295.7 & 343.1 & K134I & $9.8^{\mathrm{a}}$ & 3.06 & 2.30 & 13.65 & $11.77 \pm 0.14$ & 4 & 46 & $16.84 \pm 0.01$ & & 0.23 & \\
\hline 06331 & (6331) 1992 FZ1 & 2.36 & 0.13 & 7.77 & 129.5 & 190.0 & K134I & $5.3^{\mathrm{a}}$ & 2.65 & 1.83 & 14.23 & $12.82 \pm 0.14$ & 4 & 48 & $17.08 \pm 0.01$ & & 0.15 & \\
\hline 06503 & (6503) $1994 \mathrm{CP}$ & 2.85 & 0.04 & 2.93 & 135.4 & 264.4 & K134I & 12.9 & 2.82 & 2.04 & 14.53 & $13.21 \pm 0.43$ & 4 & 37 & $17.82 \pm 0.02$ & $7.13 \pm 0.05$ & 0.23 & 1 \\
\hline 06615 & (6615) Plutarchos & 2.17 & 0.13 & 1.80 & 129.4 & 81.2 & K134I & $3.1^{\mathrm{a}}$ & 2.15 & 1.40 & 21.27 & $14.45 \pm 0.11$ & 4 & 56 & $17.90 \pm 0.02$ & & 0.14 & \\
\hline 07120 & (7120) Davidgavine & 2.86 & 0.06 & 1.16 & 314.2 & 91.5 & K134I & 14.1 & 2.79 & 2.06 & 15.96 & $13.01 \pm 0.21$ & 4 & 55 & $17.68 \pm 0.02$ & $1.44 \pm 0.00$ & 0.19 & 1 \\
\hline 07205 & (7205) Sadanori & 2.63 & 0.12 & 1.68 & 274.6 & 337.3 & K134I & $5.3^{\mathrm{a}}$ & 2.85 & 2.13 & 15.70 & $12.81 \pm 0.26$ & 4 & 54 & $17.58 \pm 0.02$ & & 0.14 & \\
\hline 07712 & (7712) 1995 TB1 & 2.57 & 0.24 & 4.86 & 134.6 & 255.1 & K134I & $4.1^{\mathrm{a}}$ & 2.41 & 1.63 & 17.52 & $13.48 \pm 0.09$ & 4 & 54 & $17.36 \pm 0.02$ & & 0.16 & \\
\hline 08122 & (8122) Holbein & 2.38 & 0.16 & 1.54 & 302.4 & 178.8 & K134I & $3.5^{\mathrm{a}}$ & 1.99 & 1.14 & 19.30 & $14.30 \pm 0.09$ & 4 & 59 & $17.05 \pm 0.01$ & & 0.17 & \\
\hline 08434 & (8434) Columbianus & 2.97 & 0.25 & 3.13 & 118.2 & 336.6 & K134I & $10.3^{\mathrm{a}}$ & 2.26 & 1.52 & 20.36 & $13.52 \pm 0.15$ & 4 & 51 & $17.21 \pm 0.01$ & & 0.39 & \\
\hline 09072 & (9072) 1993 RX3 & 3.21 & 0.07 & 15.23 & 290.1 & 14.7 & K134I & $25.3^{\mathrm{a}}$ & 3.43 & 2.75 & 13.35 & $11.64 \pm 0.09$ & 4 & 47 & $17.30 \pm 0.01$ & & 0.15 & \\
\hline 09358 & (9358) Faro & 2.64 & 0.09 & 3.30 & 126.8 & 217.5 & K134I & $5.7^{\mathrm{a}}$ & 2.82 & 1.95 & 11.35 & $13.22 \pm 0.09$ & 4 & 43 & $17.62 \pm 0.02$ & & 0.18 & \\
\hline 09629 & (9629) Servet & 2.71 & 0.05 & 1.80 & 330.2 & 96.6 & K134I & 8.4 & 2.62 & 1.76 & 12.92 & $13.39 \pm 0.10$ & 4 & 48 & $17.47 \pm 0.02$ & & 0.20 & \\
\hline 09813 & (9813) Rozgaj & 2.27 & 0.13 & 1.62 & 201.8 & 227.3 & K134I & $3.8^{\mathrm{a}}$ & 2.07 & 1.28 & 20.88 & $14.07 \pm 0.08$ & 4 & 58 & $17.24 \pm 0.01$ & & 0.11 & \\
\hline 10361 & (10361) Bunsen & 2.29 & 0.12 & 3.64 & 143.5 & 286.4 & K134I & $2.4^{\mathrm{a}}$ & 2.09 & 1.31 & 21.08 & $14.10 \pm 0.13$ & 4 & 46 & $17.37 \pm 0.02$ & & 0.14 & \\
\hline 11181 & (11181) 1998 FG118 & 3.04 & 0.05 & 3.97 & 296.1 & 246.2 & K134I & $15.9^{\mathrm{a}}$ & 2.97 & 2.12 & 11.52 & $12.32 \pm 0.20$ & 4 & 56 & $17.01 \pm 0.01$ & & 0.40 & \\
\hline
\end{tabular}


Table 3

(Continued)

\begin{tabular}{|c|c|c|c|c|c|c|c|c|c|c|c|c|c|c|c|c|c|c|}
\hline Obj ID & Designation & $a$ & $e$ & $i$ & $\Omega$ & $\omega$ & Epoch & $D$ & $\Delta$ & $r$ & $\alpha$ & $H$ & $n$ & $m$ & $\mathrm{PTF}_{R}$ & $\begin{array}{c}\text { Period } \\
\text { (hr) }\end{array}$ & $\Delta m$ & $U$ \\
\hline 11215 & (11215) 1999 HN10 & 2.97 & 0.10 & 0.64 & 256.7 & 109.7 & K134I & 15.9 & 3.04 & 2.29 & 13.85 & $12.76 \pm 0.18$ & 4 & 54 & $17.71 \pm 0.02$ & & 0.27 & \\
\hline 11664 & (11664) Kashiwagi & 3.18 & 0.08 & 2.74 & 117.4 & 78.9 & K134I & $12.0^{\mathrm{a}}$ & 3.09 & 2.26 & 11.55 & $13.01 \pm 0.12$ & 4 & 46 & $17.93 \pm 0.03$ & & 0.25 & \\
\hline 13323 & (13323) 1998 SQ & 5.12 & 0.09 & 0.92 & 182.5 & 256.2 & K134I & $23.4^{\mathrm{a}}$ & 4.76 & 3.91 & 6.88 & $10.84 \pm 0.13$ & 4 & 61 & $17.63 \pm 0.02$ & $13.48 \pm 0.33$ & 0.17 & 1 \\
\hline 13529 & (13529) Yokaboshi & 2.24 & 0.10 & 4.36 & 312.7 & 173.6 & K134I & 4.6 & 2.03 & 1.17 & 18.41 & $13.72 \pm 0.09$ & 4 & 55 & $16.57 \pm 0.01$ & & 0.27 & \\
\hline 14973 & (14973) Rossirosina & 2.77 & 0.07 & 4.48 & 302.5 & 148.9 & K134I & 7.4 & 2.60 & 1.84 & 16.60 & $13.66 \pm 0.19$ & 4 & 38 & $17.90 \pm 0.02$ & & 0.31 & \\
\hline 16462 & (16462) $1990 \mathrm{DZ} 1$ & 2.46 & 0.09 & 5.94 & 315.7 & 180.0 & K134I & $4.4^{\mathrm{a}}$ & 2.25 & 1.41 & 17.13 & $13.53 \pm 0.34$ & 4 & 48 & $16.78 \pm 0.01$ & & 0.23 & \\
\hline 16952 & (16952) Peteschultz & 3.10 & 0.08 & 4.07 & 137.0 & 321.5 & K134I & $9.0^{\mathrm{a}}$ & 2.86 & 2.02 & 12.37 & $13.28 \pm 0.09$ & 4 & 49 & $17.84 \pm 0.02$ & & 0.22 & \\
\hline 17627 & (17627) Humptydumpty & 3.20 & 0.15 & 0.95 & 150.3 & 308.1 & K134I & 13.9 & 2.74 & 1.96 & 15.03 & $13.04 \pm 0.14$ & 4 & 45 & $17.48 \pm 0.02$ & & 0.21 & \\
\hline 18163 & (18163) Jennalewis & 2.38 & 0.14 & 5.25 & 134.6 & 313.4 & K134I & 2.9 & 2.08 & 1.27 & 19.62 & $14.71 \pm 0.20$ & 4 & 43 & $17.85 \pm 0.02$ & & 0.44 & \\
\hline 18485 & (18485) $1996 \mathrm{AB}$ & 3.19 & 0.14 & 2.54 & 298.6 & 133.4 & K134I & $13.0^{\mathrm{a}}$ & 2.88 & 2.04 & 12.46 & $13.28 \pm 0.19$ & 4 & 54 & $17.88 \pm 0.02$ & & 0.41 & \\
\hline 18947 & (18947) Cindyfulton & 2.39 & 0.14 & 2.00 & 328.1 & 156.6 & K134I & $3.4^{\mathrm{a}}$ & 2.06 & 1.17 & 16.35 & $14.94 \pm 0.35$ & 4 & 57 & $17.83 \pm 0.02$ & & 0.81 & \\
\hline 19342 & (19342) 1997 AA7 & 2.53 & 0.23 & 2.78 & 136.0 & 283.2 & K134I & 4.8 & 2.18 & 1.35 & 17.49 & $14.61 \pm 0.14$ & 4 & 55 & $17.94 \pm 0.02$ & & 0.25 & \\
\hline 19717 & (19717) 1999 UZ40 & 2.55 & 0.13 & 2.06 & 123.6 & 83.6 & K134I & 6.8 & 2.49 & 1.69 & 16.38 & $13.83 \pm 0.13$ & 4 & 31 & $17.84 \pm 0.02$ & & 0.35 & \\
\hline 20063 & (20063) 1993 RC4 & 3.22 & 0.07 & 3.98 & 313.5 & 242.6 & K134I & $11.3^{\mathrm{a}}$ & 3.13 & 2.26 & 9.97 & $12.93 \pm 0.11$ & 4 & 40 & $17.81 \pm 0.02$ & & 0.17 & \\
\hline 27716 & (27716) Nobuyuki & 2.86 & 0.24 & 6.04 & 123.9 & 359.1 & K13B4 & $6.3^{\mathrm{a}}$ & 2.17 & 1.32 & 16.76 & $13.71 \pm 0.24$ & 4 & 61 & $16.83 \pm 0.01$ & & 0.26 & \\
\hline 29514 & (29514) Karatsu & 2.94 & 0.16 & 15.31 & 293.1 & 242.9 & K134I & $10.4^{\mathrm{a}}$ & 2.63 & 1.86 & 15.93 & $13.21 \pm 0.15$ & 4 & 46 & $17.57 \pm 0.02$ & & 0.25 & \\
\hline 30872 & (30872) 1992 EM17 & 2.33 & 0.11 & 5.06 & 128.2 & 86.0 & K134I & $3.1^{\mathrm{a}}$ & 2.30 & 1.47 & 16.69 & $13.95 \pm 0.10$ & 3 & 40 & $17.47 \pm 0.02$ & & 0.09 & \\
\hline 31025 & (31025) 1996 GR & 2.30 & 0.15 & 2.69 & 97.6 & 85.3 & K134I & $2.5^{\mathrm{a}}$ & 2.06 & 1.18 & 16.50 & $15.04 \pm 0.16$ & 4 & 52 & $17.88 \pm 0.02$ & $19.20 \pm 0.52$ & 0.19 & 1 \\
\hline 56070 & (56070) 1998 YQ5 & 2.31 & 0.11 & 2.57 & 311.8 & 131.1 & K134I & $2.5^{\mathrm{a}}$ & 2.10 & 1.26 & 18.35 & $14.66 \pm 0.18$ & 4 & 53 & $17.75 \pm 0.02$ & & 0.48 & \\
\hline 69485 & (69485) $1997 \mathrm{AD}$ & 2.53 & 0.15 & 11.89 & 294.3 & 237.4 & K134I & $4.3^{\mathrm{a}}$ & 2.28 & 1.56 & 20.55 & $13.94 \pm 0.15$ & 4 & 46 & $17.69 \pm 0.02$ & & 0.27 & \\
\hline A 3557 & (103557) 2000 BK28 & 2.65 & 0.36 & 6.51 & 115.8 & 308.5 & K134I & 4.2 & 1.95 & 1.12 & 20.75 & $14.88 \pm 0.30$ & 4 & 42 & $17.50 \pm 0.02$ & & 0.41 & \\
\hline
\end{tabular}

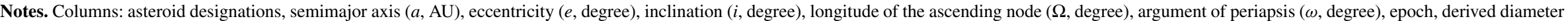

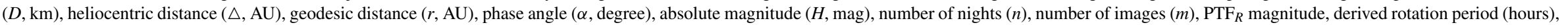
light-curve variation (mag), and rotation period quality code $(U)$.

a The WISE/NEOWISE diameter. 


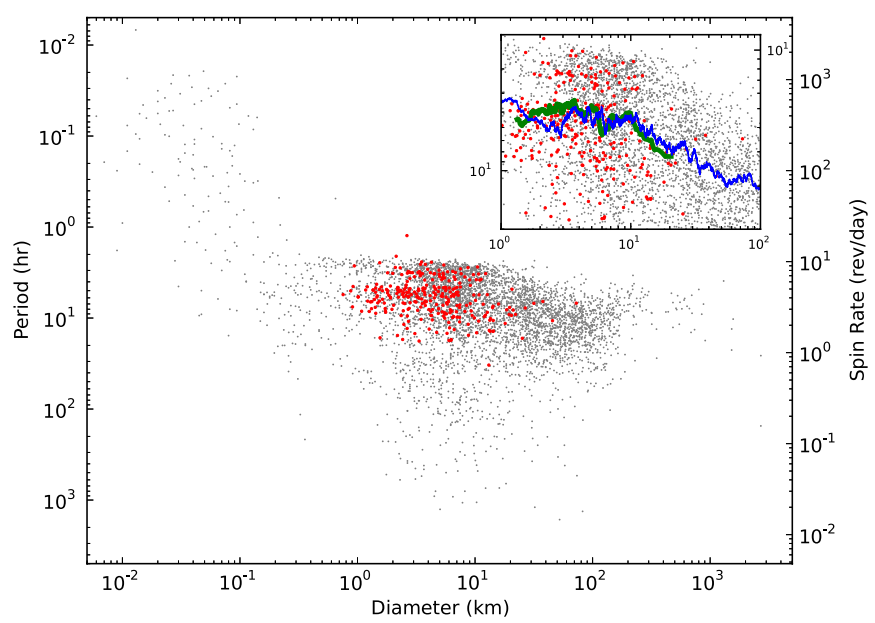

Figure 17. Plot of the diameters vs. rotation period. The red and gray filled circles are the PTF-U2 asteroids and the LCDB objects with $U \geqslant 2$, respectively. The distribution of the PTF-U2 asteroids and that of the LCDB are similar. The "spin barrier" at $\sim 2.2 \mathrm{hr}$ can obviously be seen for asteroids with diameters larger than a few hundred meters. The red filled circle above the "spin barrier" is the superfast rotator candidate, (49719) 1999 VE50. The small plot at the upper-right corner is the detailed view of the dense region, where the green and blue lines are the geometric mean spin rates of the PTF-U2 asteroids and the $\mathrm{LCDB}$, respectively.

(A color version of this figure is available in the online journal.)

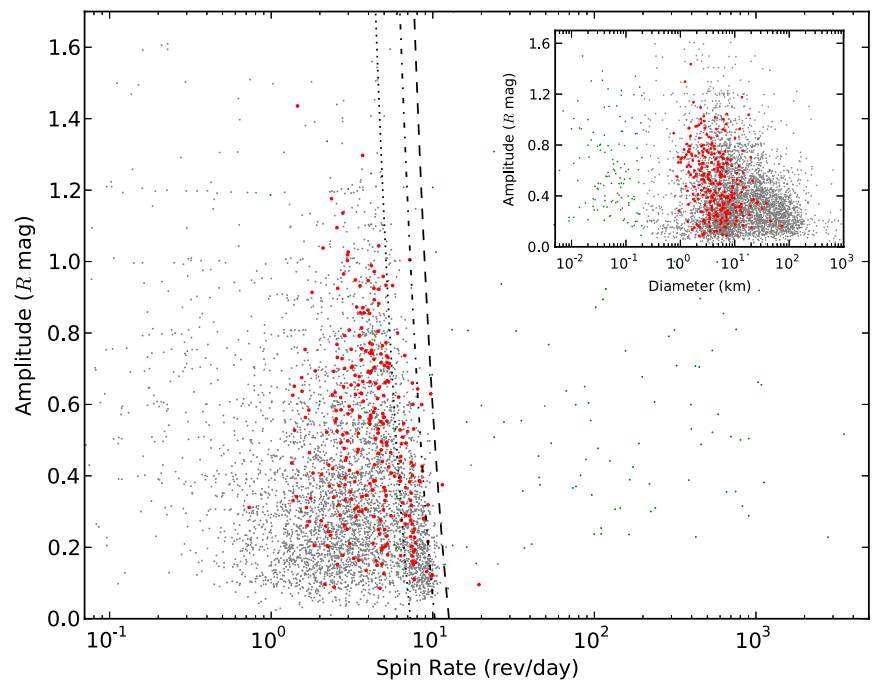

Figure 18. Plot of the spin rate vs. diameter. The red, gray, and green filled circles are the PTF-U2 asteroids, the LCDB objects with $D \geqslant 0.2 \mathrm{~km}$, and the LCDB objects with $D<0.2 \mathrm{~km}$, respectively. The dashed, dot-dashed, and dotted lines represent the spin-rate limits for "rubble pile" asteroids with bulk densities of 3, 2, and $1 \mathrm{~g} \mathrm{~cm}^{-3}$ adopted from Pravec \& Harris (2000). The small plot at the upper-right corner is the plot of the diameter vs. light-curve amplitude for the PTF-U2 asteroids.

(A color version of this figure is available in the online journal.)

bound aggregations (i.e., "rubble pile"). Several superfast rotators (i.e., rotation period $<2.2 \mathrm{hr}$ ) are located at the upper-left corner, which were usually small-sized objects and could be of a monolithic nature (Pravec \& Harris 2000). The "spin barrier" can also been seen on Figure 18, which shows the plot of the spin rate versus light-curve amplitude along with the spin rate limits for "rubble pile" asteroids with bulk densities of 3, 2, and $1 \mathrm{~g} \mathrm{~cm}^{-3}$ adopted from Pravec \& Harris (2000). One unusual object, (167714) $2001 \mathrm{OE} 84$, has a diameter of $\sim 600 \mathrm{~m}$ and a rotation period of $\sim 0.3 \mathrm{hr}$ faster than the "spin barrier," and Pravec et al. (2002) treated this object as an exceptional case. However,

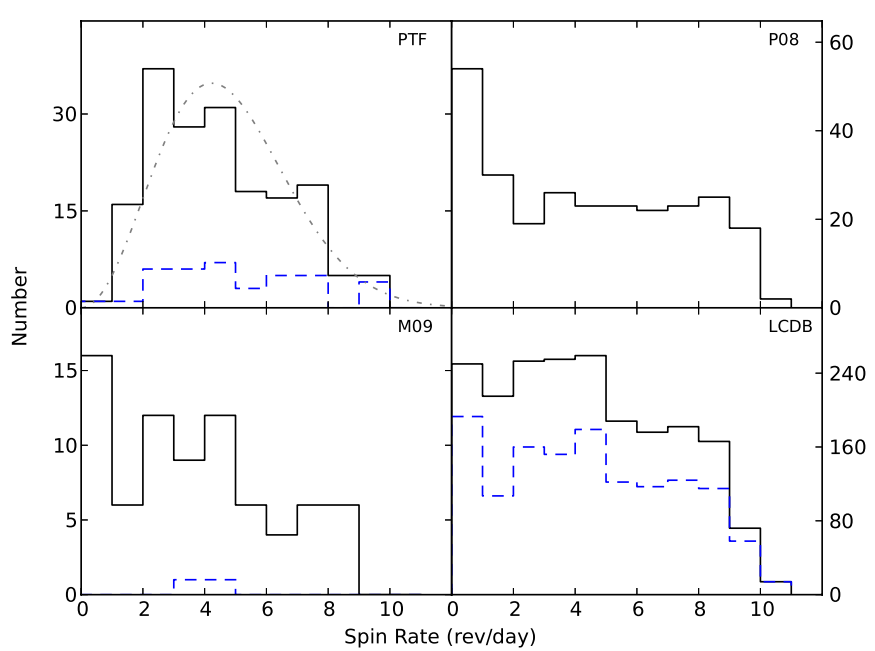

Figure 19. Distributions of spin rate of asteroids with $3<D \leqslant 15 \mathrm{~km}$ (solid lines) for the PTF-U2 asteroids (upper-left), P08 (upper-right), M09 (lowerleft), and the LCDB. The dashed lines are the asteroids with $a<2.5$ AU. The dot-dashed line on the PTF-U2 asteroids is the best-fit Maxwellian distribution.

(A color version of this figure is available in the online journal.)

Holsapple (2007) introduced a size-dependent strength asteroid model, which included tensile strength and cohesiveness in addition to gravity, to explain the existence of kilometer-sized superfast rotators. In this study, we detected one superfast rotator candidate, (49719) 1999 VE50, which has a rotation period of $1.24 \mathrm{hr}$, an amplitude of $\sim 0.29 \mathrm{mag}$, and a diameter of $\sim 2.6 \mathrm{~km}$. However, as pointed out by Harris et al. (2012), this could be a result of a random combination of scattered data points showing a relatively small light-curve amplitude. Therefore, confirming such a fast spin rate for 1999 VE50 requires a follow-up observation and detailed investigations.

The small plot at the upper-right corner of Figure 17 is the detailed view of the dense region, on which we also plot the geometric mean spin rate of the PTF-U2 asteroids and that of the LCDB by using a running box containing 30 and 100 objects, respectively. Both geometric mean spin rates begin flat and then start to decrease at $D \sim 10 \mathrm{~km}$. The decrease at $D \sim 10 \mathrm{~km}$ was considered to be a consequence of the transition from the "small" asteroids, which showed a nonMaxwellian spin-rate distribution with an excess of both fast and slow rotators, to the "large" asteroids, which showed a Maxwellian spin-rate distribution (Pravec et al. 2002). Several studies indicated that the "large" asteroid begins at the $3050 \mathrm{~km}$ diameter range (Fulchignoni et al. 1995; Donnison \& Wiper 1999; Pravec \& Harris 2000), but we were not able to determine where the "large" asteroids begin due to only a few objects with $D>30 \mathrm{~km}$ in the PTF-U2 asteroids.

The spin rate distribution of the "small" asteroids also provided valuable information to understand asteroid spin rate evolution. In order to compare with the result of Pravec et al. (2008, P08 hereafter), we selected asteroids with $3 \mathrm{~km}<D \leqslant$ $15 \mathrm{~km}$ from the PTF-U2 asteroids (Masiero et al. 2009, M09 hereafter) and the LCDB to generate spin-rate distributions in Figure 19. In contrast to the flat distribution of P08, the other three showed a number decrease at the spin rate of $>5 \mathrm{rev} \mathrm{day}^{-1}$. Warner et al. (2009) pointed out that the number decrease was an observational bias due to a tendency toward smaller light-curve amplitude with increasing spin rates (see Figure 18), while M09 showed that the multiple YORP-braking stages that existed in main belt asteroids could lead to such a result. However, M09 

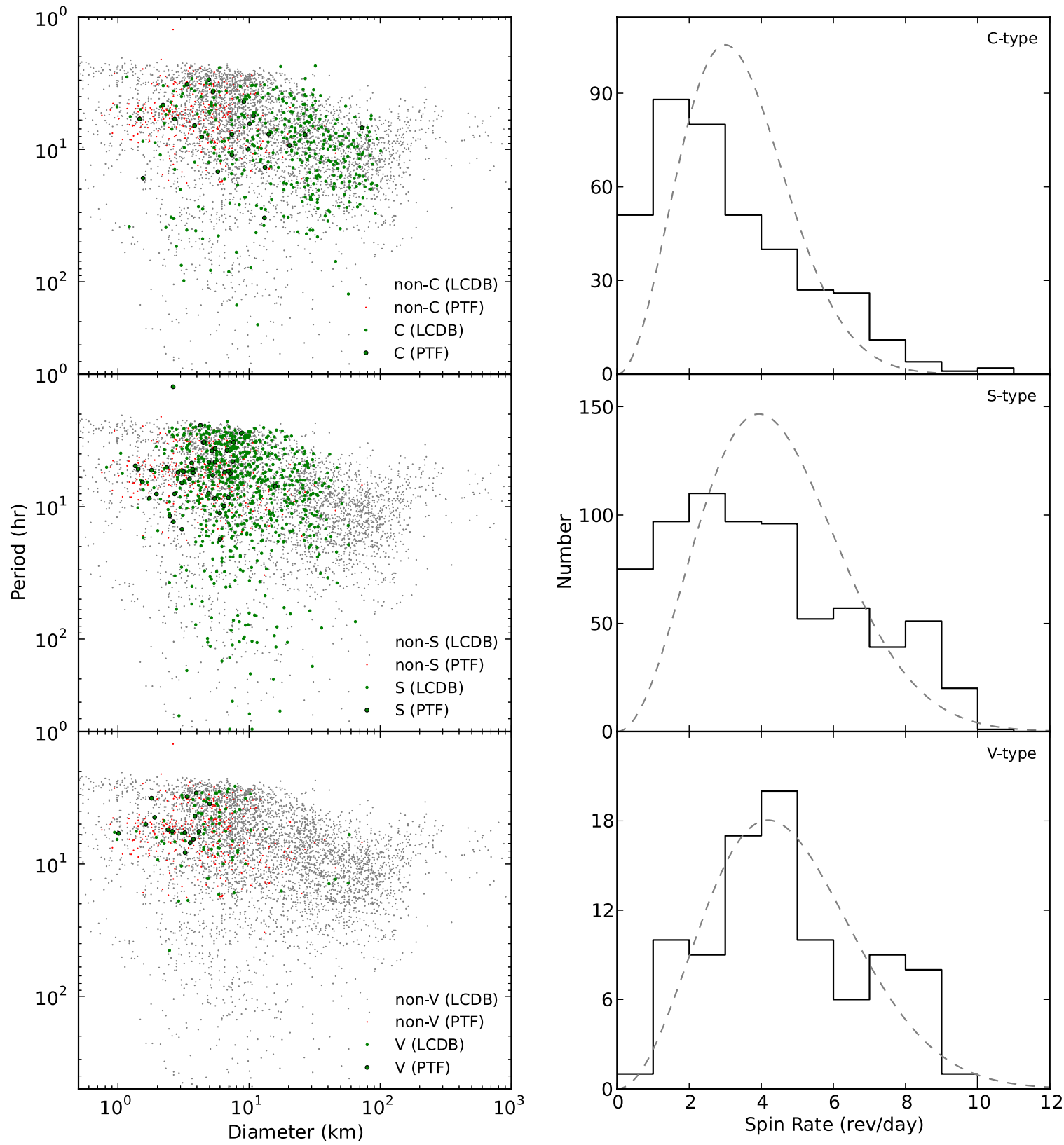

Figure 20. Left column: the plots of the diameter vs. rotation period for C- (upper panel), S- (middle panel), and V-type (lower panel) asteroids. The bigger green filled circles with black edge and the smaller green filled circles represent PTF-U2 asteroids and the LCDB objects with available taxonomy, respectively. The red and gray dots are the PTF-U2 asteroids and LCDB objects other than the corresponding taxonomic type in each plot, respectively. The taxonomic types are obtained from SDSS colors. Right column: the distributions of spin rate (solid lines) for C- (upper panel), S- (middle panel), and V-type (lower panel) asteroids. The dashed line on each panel is the best-fit Maxwellian distribution. The samples include both the PTF-U2 asteroids and the LCDB objects.

(A color version of this figure is available in the online journal.)

also mentioned that the discrepancy between P08 and the others could be the result of different survey methods (i.e., the PTF, M09, and the LCDB are untargeted surveys or collecting data sets, whereas P08 targets individual asteroids with $a<2.5 \mathrm{AU})$. If we restrict the comparison to the asteroids with $a<2.5 \mathrm{AU}$, regardless of only two samples in M09, the number decrease at the spin rate of $>5 \mathrm{rev}$ day $^{-1}$ still showed on the PTF-U2 asteroids and the LCDB (see the dashed lines in Figure 19). Therefore, a large sample of asteroid rotation period from a single survey like the PTF is of paramount importance to reveal the spin-rate distribution of the "small" asteroids. The best-fit Maxwellian distribution for the PTF-U2 asteroids is also shown in Figure 19. We see that the PTF-U2 asteroids deviate somewhat to the Maxwellian form, which means that mechanisms other than collision are involved in asteroid spin-rate evolution as well.

To gain an approximate idea of how the spin rate distributes for different taxonomic-type asteroids, we summarized the plots of the diameter versus rotation period and the distributions of spin rate for available S-, C-, and V-type asteroids in Figure 20. The taxonomic types were determined by using SDSS color (Parker et al. 2008). We were not able to tell the scatter plots from one another by eye (the left column in Figure 20); however, the spin-rate distributions showed some difference (the right column in Figure 20). The C-type distribution showed a decreasing number with increasing spin rate, the S-type distribution had 
a number drop at a spin rate of $>5 \mathrm{rev} \mathrm{day}^{-1}$, and the V-type distribution demonstrated a number enhancement around a spin rate of $65 \mathrm{rev} \mathrm{day}^{-1}$. Moreover, none of them could be fitted reasonably by a Maxwellian distribution. When we ran the twosample Kolmogorov-Smirnov (KS) test for each pair of these three types of asteroids to compare their spin-rate distributions, all of the KS test $p$ values were much lower than 0.005 . Although this indicates that these three different types were unlikely to come from the same population, we believed it could be greatly affected by the insufficient number and incompleteness in our samples, especially the different diameter ranges of these three subgroups (see the left column in Figure 20). Therefore, we expect to have a more accurate comparison on this topic when more asteroid rotation periods are available from observations now in planning.

The asteroid light-curve profile is a powerful tool to probe asteroid shape as well as to discover binary asteroids. The inset of Figure 18 shows the plot of diameter versus light-curve amplitude. Both the PTF-U2 asteroids and the LDCB show a boundary from the upper middle to the lower right that indicates that relatively large asteroids tend to have small light-curve amplitudes. Most PTF-U2 asteroids show simple sinusoidallike folded light curves. However, we found two binary asteroid candidates, (7452) Izabelyuria and (75640) 2000 AE55 (see Figure 15), whose light curves showed a deep V-shaped minima and an inverse U-shaped maxima with a relatively long rotation period (Pravec et al. 2006). The fractions of binary asteroid in different asteroid groups provide important constraints on the binary asteroid formation models. Therefore, an overall survey on asteroid binary population with large samples could reveal a clearer picture on how different mechanisms work on binary asteroid formation.

\section{SUMMARY AND DISCUSSION}

This study has demonstrated the capability of the PTF to pursue large numbers of asteroid rotation periods via a survey of 12 PTF fields in four consecutive nights of observation with a cadence of $\sim 20$ minutes during 2013 February 15-18. The PTF photometric data were used to extract the light curves of known asteroids and measure their rotation periods. There were 2500 known asteroids with more than five detections, and 312 of them had highly reliable rotation periods in this work. The plots of the spin rate versus diameter for the PTF-U2 asteroids and the LCDB were very similar. Both show the "spin barrier" clearly at $\sim 2.2 \mathrm{hr}$ and similar geometric mean spin rates. The spin-rate distribution of the PTF-U2 asteroids showed a number decrease at the spin rate of $>5 \mathrm{rev} \mathrm{day}^{-1}$, which is not a Maxwellian distribution nor as flat as shown by Pravec et al. (2008). The non-Maxwellian distribution indicated that the asteroid spinrate evolution was not only affected by collisions but also by other mechanics, in particular the YORP effect. The rough test for available S-, C-, and V-type asteroids does not yield a clear conclusion on any difference between the spin-rate distributions of various taxonomic types. However, we did find one superfast rotator candidate, (49719) 1999 VE50, which had a rotation period of $1.24 \mathrm{hr}$ and a diameter of $\sim 2.6 \mathrm{~km}$. If the fast spin rate of 1999 VE50 is confirmed, the size-dependent strength asteroid model will be supported. In addition, we also detected two binary asteroid candidates, (7452) Izabelyuria and (75640) 2000 AE55. The tendency toward smaller light-curve amplitudes with increasing diameter is seen in the PTF-U2 asteroids as well, which means that large asteroids tend to have rounder shapes.
Because our target fields are close to the Galactic plane toward the anti-Galactic center direction, the detection rate of known asteroids is expected to be lower than that in the off-Galactic plane fields, as will the number of highly reliable rotation periods. For an approximate estimation, it is very likely to obtain 10,000 asteroid rotation periods by reproducing this kind of observation around 20-30 times. In addition, we also expect to retrieve more asteroid rotation periods from previously observed high-cadence fields, which are now under analysis. Such a huge amount of asteroid rotation periods can provide more definite constraints on various studies, such as how different mechanisms (e.g., collision, YORP effect, and tidal force during their encounter with planets) are involved in asteroid spin-rate evolution, and how tensile strength and cohesiveness account for the spin-rate limits of different-sized asteroids (i.e., the size-dependent strength for asteroids; Holsapple 2007) and the survey on an asteroid light-curve profile (i.e., asteroid shape/ axis ratio). We also can further investigate the asteroid spin rate as a function of the size, taxonomy, dynamical group, and asteroid family. In addition, more binary asteroids will be discovered to reveal their fractions in different asteroid populations and provide important constraints on their formation models.

This work is supported in part by the National Science Council of Taiwan under the grants NSC 101-2119-M-008007-MY3 and NSC 102-2112-M-008-019-MY3. We thank Eran Ofek for valuable comments and suggestions that made the manuscript more complete. We also thank the referee, Joseph Masiero, for useful comments that helped to improve the content of the paper. This publication makes use of data products from WISE, which is a joint project of the University of California, Los Angeles, and the Jet Propulsion Laboratory/ California Institute of Technology, funded by the National Aeronautics and Space Administration. This publication also makes use of data products from NEOWISE, which is a project of the Jet Propulsion Laboratory/California Institute of Technology, funded by the Planetary Science Division of the National Aeronautics and Space Administration. We gratefully acknowledge the extraordinary services specific to NEOWISE contributed by the International Astronomical Unions Minor Planet Center, operated by the Harvard-Smithsonian Center for Astrophysics, and the Central Bureau for Astronomical Telegrams, operated by Harvard University.

\section{REFERENCES}

Agüeros, M. A., Covey, K. R., Lemonias, J. J., et al. 2011, ApJ, 740, 110 Bertin, E., \& Arnouts, S. 1996, A\&AS, 117, 393

Bowell, E., Hapke, B., Domingue, D., et al. 1989, Asteroids II (Tucson, AZ: Univ. Arizona Press), 524

Donnison, J. R., \& Wiper, M. P. 1999, MNRAS, 302, 75

Fulchignoni, M., Barucci, M. A., di Martino, M., \& Dotto, E. 1995, A\&A, 299, 929

Grav, T., Mainzer, A. K., Bauer, J., et al. 2011, ApJ, 742, 40

Grillmair, C. J., Laher, R., Surace, J., et al. 2010, in ASP Conf. Ser. 434, Astronomical Data Analysis Software and Systems XIX, ed. Y. Mizumoto, K.-I. Morita, \& M. Ohishi (San Francisco, CA: ASP), 28

Harris, A. W. 1996, LPSC, 27, 493

Harris, A. W., Pravec, P., \& Warner, B. D. 2012, Icar, 221, 226

Hergenrother, C. W., \& Whiteley, R. J. 2011, Icar, 214, 194

Holsapple, K. A. 2007, Icar, 187, 500

Jewitt, D., Ishiguro, M., \& Agarwal, J. 2013, ApJL, 764, L5

Laher, R. R., Surace, J., Grillmair, C. J., et al. 2014, PASP, submitted (arXiv:1404.1953)

Law, N. M., Dekany, R. G., Rahmer, G., et al. 2010, Proc. SPIE, 7735, 77353M Law, N. M., Kulkarni, S. R., Dekany, R. G., et al. 2009, PASP, 121, 1395 
Levitan, D., Fulton, B. J., Groot, P. J., et al. 2011, ApJ, 739, 68 Mainzer, A., Grav, T., Bauer, J., et al. 2011, ApJ, 743, 156

Masiero, J., Jedicke, R., Durech, J., et al. 2009, Icar, 204, 145

Masiero, J. R., Mainzer, A. K., Grav, T., et al. 2011, ApJ, 741, 68

Ofek, E. O., Frail, D. A., Breslauer, B., et al. 2011, ApJ, 740, 65

Ofek, E. O., Laher, R., Law, N., et al. 2012a, PASP, 124, 62

Ofek, E. O., Laher, R., Surace, J., et al. 2012b, PASP, 124, 854

Parker, A., Ivezić, Ž., Jurić, M., et al. 2008, Icar, 198, 138

Pilcher, F., Benishek, V., \& Krajewski, R. 2009, MPBu, 36, 40

Polishook, D., \& Brosch, N. 2008, Icar, 194, 111

Polishook, D., Ofek, E. O., Waszczak, A., et al. 2012, MNRAS, 421, 2094

Pravec, P., \& Harris, A. W. 2000, Icar, 148, 12
Pravec, P., Harris, A. W., Vokrouhlický, D., et al. 2008, Icar, 197, 497

Pravec, P., Kušnirák, P., Šarounová, L., et al. 2002, in Asteroids, Comets, and Meteors: ACM 2002, ed. B. Warmbein (ESA SP-500: Noordwijk: European Space Agency), 743

Pravec, P., Scheirich, P., Kušnirák, P., et al. 2006, Icar, 181, 63

Rau, A., Kulkarni, S. R., Law, N. M., et al. 2009, PASP, 121, 1334

Rubincam, D. P. 2000, Icar, 148, 2

Salo, H. 1987, Icar, 70, 37

Tedesco, E. F., Cellino, A., \& Zappalá, V. 2005, AJ, 129, 2869

Warner, B. D., Harris, A. W., \& Pravec, P. 2009, Icar, 202, 134

York, D. G., Adelman, J., Anderson, J. E., Jr., et al. 2000, AJ, 120, 1579 